\title{
QUALITATIVE PROPERTIES OF SADDLE-SHAPED SOLUTIONS TO BISTABLE DIFFUSION EQUATIONS
}

\author{
X. CABRÉ AND J. TERRA
}

\begin{abstract}
We consider the elliptic equation $-\Delta u=f(u)$ in the whole $\mathbb{R}^{2 m}$, where $f$ is of bistable type. It is known that there exists a saddleshaped solution in $\mathbb{R}^{2 m}$. This is a solution which changes sign in $\mathbb{R}^{2 m}$ and vanishes only on the Simons cone $\mathcal{C}=\left\{\left(x^{1}, x^{2}\right) \in \mathbb{R}^{m} \times \mathbb{R}^{m}:\left|x^{1}\right|=\left|x^{2}\right|\right\}$. It is also known that these solutions are unstable in dimensions 2 and 4 .

In this article we establish that when $2 m=6$ every saddle-shaped solution is unstable outside of every compact set and, as a consequence has infinite Morse index. For this we establish the asymptotic behavior of saddle-shaped solutions at infinity. Moreover we prove the existence of a minimal and a maximal saddle-shaped solutions and derive monotonicity properties for the maximal solution.

These results are relevant in connection with a conjecture of De Giorgi on 1D symmetry of certain solutions. Saddle-shaped solutions are the simplest candidates, besides $1 \mathrm{D}$ solutions, to be global minimizers in high dimensions, a property not yet established.
\end{abstract}

\section{INTRODUCTION AND MAIN RESULTS}

This paper concerns saddle-shaped solutions to bistable diffusion equations

$$
-\Delta u=f(u) \quad \text { in } \mathbb{R}^{n}
$$

where $n=2 m$ is an even integer. It is the follow-up to our previous article [14]. Here, we study qualitative properties of saddle-shaped solutions, such as their stability, asymptotic behavior, and monotonicity properties.

Our interest on these solutions originates from a conjecture raised by De Giorgi [17] in 1978. It consists of establishing whether every bounded solution $u$ of the Allen-Cahn equation

$$
-\Delta u=u-u^{3} \quad \text { in } \mathbb{R}^{n}
$$

which is monotone in one direction (say, for instance $\partial_{x_{n}} u>0$ in $\mathbb{R}^{n}$ ), depends only on one Euclidean variable (equivalently, all its level sets are hyperplanes), at least if $n \leq 8$. The conjecture has been proven to be true when the dimension $n=2$ by Ghoussoub and Gui [22], and when $n=3$

Both authors were supported by the Spain Research projects MTM2005-07660-C02-01 and MTM2008-06349-C03-01.

The second author was supported by the FCT grant SFRH/BD/8985/2002. 
by Ambrosio and Cabré [5]. For $4 \leq n \leq 8$ and assuming the additional condition

$$
\lim _{x_{n} \rightarrow \pm \infty} u\left(x^{\prime}, x_{n}\right)= \pm 1 \quad \text { for all } x^{\prime} \in \mathbb{R}^{n-1},
$$

it has been established by Savin [26]. Recently, del Pino, Kowalczyk, and Wei [20] have announced that the statement of the conjecture does not hold for $n \geq 9$-as suggested in De Giorgi's original statement. In addition, the monotone and non-flat solution that they construct satisfies (1.3). However, for $4 \leq n \leq 8$ the conjecture in its original statement is still open, and to our knowledge no clear evidence is known about its validity or not. That is:

Open Question 1. For $4 \leq n \leq 8$, does the conjecture hold in its original statement, that is, without assuming the limiting condition (1.3)?

Next, we explain how assumption (1.3) enters in the proof of Savin's result, and we state another version of the conjecture (Open Question 2 below). This new version will lead to the study of saddle-shaped solutions and to an open problem possibly easier (or more natural) than Open Question 1 above. First, recall the following result.

Theorem 1.1 (Alberti-Ambrosio-Cabré 2]). Suppose that $u$ is a solution of (1.2) satisfying $\partial_{x_{n}} u>0$ in $\mathbb{R}^{n}$ and the condition (1.3) on limits. Then, $u$ is a global minimizer in $\mathbb{R}^{n}$. That is,

$$
\mathcal{E}(u, \Omega) \leq \mathcal{E}(u+\xi, \Omega)
$$

for every bounded domain $\Omega$ and every $C^{\infty}$ function $\xi$ with compact support in $\Omega$. Here, $\mathcal{E}$ denotes the energy functional associated to (1.2).

Recall that the energy functional associated to equation (1.1) is

$$
\mathcal{E}(v, \Omega):=\int_{\Omega}\left\{\frac{1}{2}|\nabla v|^{2}+G(v)\right\} d x, \quad \text { where } G^{\prime}=-f .
$$

See 2 for the original proof of the Theorem 1.1 (which was quite involved and used calibrations), and section 3 of [26] for a simple proof due to L. Caffarelli. Now we can state a deep result of Savin [26].

Theorem 1.2 (Savin [26]). Assume that $n \leq 7$ and that $u$ is a global minimizer of (1.2) in $\mathbb{R}^{n}$. Then, the level sets of $u$ are hyperplanes.

Note that this result makes no assumptions on the monotonicity or the limits at infinity of the solution. Now, Savin's result on monotone solutions needs to assume (1.3) only to guarantee, by Theorem 1.1, that the solution is actually a global minimizer. Then, Theorem 1.2 (and the gain of one dimension $n=8$ thanks to the monotonicity of the solution) leads to Savin's result on monotone solutions with limits \pm 1 .

Again, Theorem 1.2 makes no additional assumption on the solutions (minimizers here). It establishes that in dimensions $n \leq 7$, $1 \mathrm{D}$ solutions (i.e., solutions depending only on one Euclidean variable) are the only global minimizers of (1.2). In addition, the hypothesis $n \leq 7$ on its statement is 
believed to be sharp -we will explain this later in more detail. That is, in $\mathbb{R}^{8}$ one expects the existence of a global minimizer which is not $1 \mathrm{D}$.

Open Question 2. Is there a global minimizer of $(1.2)$ in $\mathbb{R}^{8}$ whose level sets are not hyperplanes? Related to this, it will be natural to ask the following. Are saddle-shaped solutions (as defined below) global minimizers of $(1.2)$ in $\mathbb{R}^{8}$, or at least in higher even dimensions?

A positive answer to this last question would give an alternative way to that of [20] to prove the existence of a counter-example of the conjecture of De Giorgi in $\mathbb{R}^{9}$. Indeed, saddle-shaped solutions are even functions of each coordinate $x_{i}$. Thus, by a result of Jerison and Monneau [24] (Theorem 2.3 below, in the next section), if a saddle solution were a global minimizer in $\mathbb{R}^{2 m}$, then the conjecture of De Giorgi on monotone solutions would not hold in $\mathbb{R}^{2 m+1}$.

Let us explain why dimension $n=8$, and also saddle-shaped solutions, play an important role. By a connection of variational nature between equation (1.2) and the theory of minimal surfaces (see [2, 24, 26]), every level set of a global minimizer should converge at infinity to the boundary of a minimal set - minimal here in in the variational sense, that is, minimizing perimeter. See [26] for precise statements. Now, a deep theorem (mostly due to Simons [28]; see Theorem 17.3 of [23]) states that the boundary of a minimal set in all of $\mathbb{R}^{n}$ must be a hyperplane if $n \leq 7$. Instead, in $\mathbb{R}^{8}$ and higher dimensions, there exist minimal sets different than half-spaces. The simplest example is the Simons cone, as proved by Bombieri-De Giorgi-Giusti [13].

The Simons cone is defined by

$$
\mathcal{C}=\left\{x \in \mathbb{R}^{2 m}: x_{1}^{2}+x_{2}^{2}+\cdots+x_{m}^{2}=x_{m+1}^{2}+x_{m+2}^{2}+\cdots+x_{2 m}^{2}\right\}
$$

It is easy to verify that $\mathcal{C}$ has zero mean curvature at every $x \in \mathcal{C} \backslash\{0\}$, in every dimension $2 m \geq 2$. However, it is only in dimensions $2 m \geq 8$ that $\mathcal{C}$ is in addition a minimizer of the area functional, i.e., it is a minimal cone in the variational sense. For all these questions, see the book of Giusti [23]. The recent paper [18] contains a short proof of the minimality of the Simons cone when $2 m \geq 8$. Later in this introduction we will also make some comments on the Morse index of the Simons cone depending on the dimension.

Let us also mention here that for another variational problem (a one-phase free boundary problem for harmonic functions), a similar program has been undertaken by Caffarelli-Jerison-Kenig [15] and De Silva-Jerison [19]. They have established, respectively, the smoothness of minimizers in dimension $n \leq 3$ and the existence of a non-smooth global minimizer in $\mathbb{R}^{7}$ - the dimensions in between being still an open question.

Saddle-shaped solutions to the bistable diffusion equation are closely related to the Simons cone, as follows. For $x=\left(x_{1}, \ldots, x_{2 m}\right) \in \mathbb{R}^{2 m}$, let us 
define two radial variables $s$ and $t$ by

$$
\left\{\begin{array}{l}
s=\sqrt{x_{1}^{2}+\ldots+x_{m}^{2}} \geq 0 \\
t=\sqrt{x_{m+1}^{2}+\ldots+x_{2 m}^{2}} \geq 0 .
\end{array}\right.
$$

The Simons cone is given by

$$
\mathcal{C}=\{s=t\}=\partial \mathcal{O}, \quad \text { where } \mathcal{O}=\{s>t\} .
$$

The following is the notion of saddle solution, which we introduced in [14].

Definition 1.3. Let $f \in C^{1}(\mathbb{R})$ be odd. We say that $u: \mathbb{R}^{2 m} \rightarrow \mathbb{R}$ is a saddle-shaped solution (or simply a saddle solution) of

$$
-\Delta u=f(u) \quad \text { in } \mathbb{R}^{2 m}
$$

if $u$ is a bounded solution of (1.7) and, with $s$ and $t$ defined by (1.6),

(a) $u$ depends only on the variables $s$ and $t$. We write $u=u(s, t)$;

(b) $u>0$ in $\mathcal{O}:=\{s>t\}$;

(c) $u(s, t)=-u(t, s)$ in $\mathbb{R}^{2 m}$.

Saddle-shaped solutions should be relevant in connection with Theorem 1.2 and Open Question 2 above on minimizers of the bistable diffusion equation due to the different variational properties of their zero level set (the Simons cone) depending on the dimension - together with the connection between the diffusion equation and minimal surfaces. Note also that saddle solutions are even with respect to each coordinate $x_{i}, 1 \leq i \leq 2 m$, as in the result of Jerison-Monneau - Theorem 2.3 below, in section 2 .

On the other hand, the conjecture of De Giorgi and Open Question 1 on monotone solutions are related to minimal graphs - instead of minimal cones or minimal sets. The existence of minimal graphs (of functions $\varphi: \mathbb{R}^{k} \rightarrow \mathbb{R}$ ) different than hyperplanes is also well understood. They exist only when the dimension $k \geq 8$. The simplest one was built by Bombieri-De GiorgiGiusti [13] for $k=8$ and has the Simons cone as zero level set. This minimal graph (living in $\mathbb{R}^{9}$ ) is used in [20] to construct the counter-example to the conjecture of De Giorgi in $\mathbb{R}^{9}$. Note that the Simons cone in $\mathbb{R}^{8}$ is a variety of dimension 7 , while the previous graph is of dimension 8 .

Towards the complete understanding and characterization of global minimizers (see Open Question 2), we study saddle-shaped solutions and their qualitative properties. To state our precise results, given a $C^{1}$ nonlinearity $f: \mathbb{R} \rightarrow \mathbb{R}$ and $M>0$, define

$$
G(u)=\int_{u}^{M} f
$$

We have that $G \in C^{2}(\mathbb{R})$ and $G^{\prime}=-f$. For some $M>0$, and with $G$ defined as above, we assume that

$$
\left\{\begin{array}{l}
f \text { is odd in } \mathbb{R} \\
G \geq 0=G( \pm M) \text { in } \mathbb{R} \text { and } G>0 \text { in }(-M, M) \\
f^{\prime} \text { is decreasing in }(0, M) .
\end{array}\right.
$$


In Section 2 we comment further these hypothese on $f$. They are satisfied by $f(u)=u-u^{3}$, for which $G(u)=(1 / 4)\left(1-u^{2}\right)^{2}$ and $M=1$.

In [14 we defined saddle-shaped solutions as above and proved their existence in all even dimensions. Namely, we proved:

Theorem $1.4\left([14)\right.$. Let $f \in C^{1}(\mathbb{R})$ satisfy conditions (1.9) for some constant $M>0$, where $G$ is defined by (1.8). Then, for every even dimension $2 m \geq 2$, there exists a saddle-shaped solution $u \in C^{2}\left(\mathbb{R}^{2 m}\right)$ of $-\Delta u=f(u)$ in $\mathbb{R}^{2 m}$, as in Definition 1.3 .

Saddle solutions were first studied by Dang, Fife, and Peletier [16 in dimension $n=2$ for $f$ odd, bistable, and with $f(u) / u$ decreasing for $u \in(0,1)$. They proved the existence and uniqueness of a saddle solution in dimension 2. They also established monotonicity properties and the asymptotic behavior at infinity of the saddle solution. Its instability (see Definition 1.5 below), already indicated in a partial result of [16], was studied in detail by Schatzman [27] by analysing the linearized operator at the saddle solution and showing that, when $f(u)=u-u^{3}$, it has exactly one negative eigenvalue. That is, the saddle solution of the Allen-Cahn equation in dimension 2 has Morse index 1; see Definition 1.8 below.

The precise notion of stability or instability that we use is the following.

Definition 1.5. Let $f \in C^{1}(\mathbb{R})$. We say that a bounded solution $u$ of (1.1) is stable if the second variation of energy $\delta^{2} \mathcal{E} / \delta^{2} \xi$ with respect to compactly supported perturbations $\xi$ is nonnegative. That is, if

$$
Q_{u}(\xi):=\int_{\mathbb{R}^{n}}\left\{|\nabla \xi|^{2}-f^{\prime}(u) \xi^{2}\right\} d x \geq 0 \quad \text { for all } \xi \in C_{c}^{\infty}\left(\mathbb{R}^{n}\right) .
$$

We say that $u$ is unstable if and only if $u$ is not stable.

Clearly, every global minimizer (as defined in Theorem 1.1) is a stable solution.

The instability of the saddle solution in dimension 2 (in the sense of Definition 1.5) is nowadays a consequence of a more recent result related to the conjecture of De Giorgi. Namely, 22 and 9 established that, for all $f \in C^{1}$, every bounded stable solution of (1.1) in $\mathbb{R}^{2}$ must be a 1D solution, that is, a solution depending only on one Euclidean variable. In particular, the saddle-shaped solution in $\mathbb{R}^{2}$ can not be stable.

In [14] we established the instability outside of every compact set of saddle solutions in dimension 4 and, as a consequence, their infinite Morse index (see Definition 1.8 below). In this paper we establish this same result in dimension 6. In addition, the computations in the last section suggest the possibility of saddle solutions being stable in dimensions $2 m \geq 8$. Such stability result would be a promising hint towards the possible global minimality of saddle solutions in high dimensions, and hence towards a construction of a counter-example to the conjecture of De Giorgi through the method of Jerison-Monneau [24]. 
The proof of our result in dimension 6 uses two new ingredients of independent interest, which hold in any dimension. The first concerns the asymptotic behavior of saddle solutions at infinity. The second one establishes the existence of a minimal and a maximal saddle solutions, as well as some key monotonicity properties of the maximal saddle solution.

Note that for functions $u$ depending only on $s$ and $t$, such as saddle solutions, the energy functional (1.4) becomes

$$
\mathcal{E}(u, \Omega)=c_{m} \int_{\Omega} s^{m-1} t^{m-1}\left\{\frac{1}{2}\left(u_{s}^{2}+u_{t}^{2}\right)+G(u)\right\} d s d t,
$$

where $c_{m}$ is a positive constant depending only on $m$ - here we have assumed that $\Omega \subset \mathbb{R}^{2 m}$ is radially symmetric in the first $m$ variables and also in the last $m$ variables, and we have abused notation by identifying $\Omega$ with its projection in the $(s, t)$ plane. In these variables, the semilinear equation (1.7) reads

$$
-\left(u_{s s}+u_{t t}\right)-(m-1)\left(\frac{u_{s}}{s}+\frac{u_{t}}{t}\right)=f(u) \quad \text { for } s>0, t>0 .
$$

The proof of the instability theorem in dimension 4 relied strongly on the following estimate that we established in [14] (see also Proposition 2.2 in section 2). It states that

$$
|u(x)| \leq\left|u_{0}\left(\frac{s-t}{\sqrt{2}}\right)\right| \quad \text { for all } x \in \mathbb{R}^{2 m}
$$

and for every saddle solution $u$, where $u_{0}$ is the monotone solution of $-u^{\prime \prime}=$ $f(u)$ in $\mathbb{R}$ vanishing at 0 . The quantity $|s-t| / \sqrt{2}$ turns out to be the distance to the cone $\mathcal{C}$. This result suggests a new change of variables. Namely we define

$$
\left\{\begin{array}{l}
y=(s+t) / \sqrt{2} \\
z=(s-t) / \sqrt{2}
\end{array}\right.
$$

which satisfy $y \geq 0$ and $-y \leq z \leq y$. Note that $|z|$ is the distance of any point $x \in \mathbb{R}^{2 m}$ to the Simons cone $\mathcal{C}$; thus, we have $\mathcal{C}=\{z=0\}$ (see Figure 11).

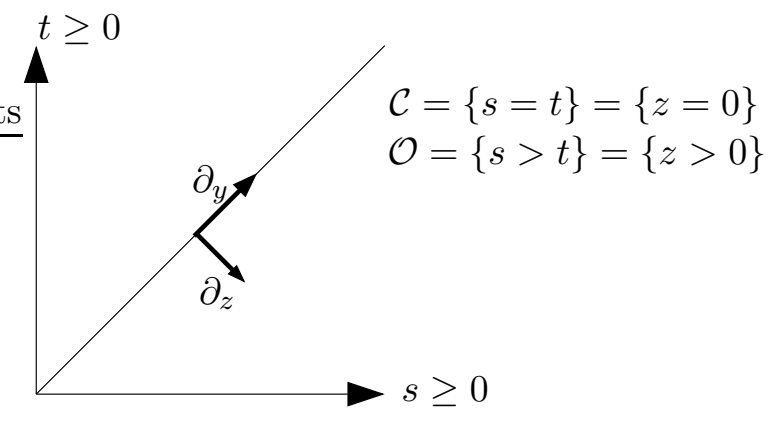

FIGURE 1. 
With these new variables estimate (1.13) may be written as

$$
|u(x)| \leq\left|u_{0}(z)\right| \quad \text { for all } x \in \mathbb{R}^{2 m} .
$$

If we take into account these variables, equation (1.7) becomes

$$
u_{y y}+u_{z z}+\frac{2(m-1)}{y^{2}-z^{2}}\left(y u_{y}-z u_{z}\right)+f(u)=0 .
$$

Using estimate (1.13), instability in dimension 4 follows from establishing that the quadratic form $Q$ defined by (1.10) with the solution $u$ replaced by the explicit function $u_{0}((s-t) / \sqrt{2})$, is negative when $n=4$ for some test function $\xi$. By (1.13), this implies that $Q_{u}$ is also negative for some test function, where $u$ is any given solution vanishing on $\mathcal{C}$. Hence, $u$ is unstable when $n=4$.

This proof can not be generalized to dimension 6 as it turns out that $u_{0}((s-t) / \sqrt{2})$ is, in some sense, asymptotically stable at infinity for perturbations with separate variables in $(y, z)$.

Hence, the proof of instability in dimension 6 requires a more precise argument. We use the equation satisfied by $\bar{u}_{z}$, where $\bar{u}$ is a maximal saddle solution constructed below, as well as some monotonicity and asymptotic properties of $\bar{u}$ established in this article and stated in the next two theorems.

The following are the new results needed in our proof of instability of saddle solutions in dimension 6 . The two results are actually important by themselves. The first one concerns the asymptotic behavior at infinity for a class of solutions which contains saddle solutions - and also other possible solutions not depending only on $s$ and $t$.

Theorem 1.6. Let $f$ satisfy conditions (1.9) and let $u$ be a bounded solution of $-\Delta u=f(u)$ in $\mathbb{R}^{2 m}$ such that $u \equiv 0$ on $\mathcal{C}, u>0$ in $\mathcal{O}=\{s>t\}$ and $u$ is odd with respect to $\mathcal{C}$. Then, denoting

$$
U(x):=u_{0}((s-t) / \sqrt{2})=u_{0}(z) \quad \text { for } x \in \mathbb{R}^{2 m},
$$

we have

$$
(u-U)(x) \rightarrow 0 \quad \text { and } \quad(\nabla u-\nabla U)(x) \rightarrow 0,
$$

uniformly as $|x| \rightarrow \infty$. That is,

$$
\|u-U\|_{L^{\infty}\left(\mathbb{R}^{2 m} \backslash B_{R}\right)}+\|\nabla u-\nabla U\|_{L^{\infty}\left(\mathbb{R}^{2 m} \backslash B_{R}\right)} \rightarrow 0 \text { as } R \rightarrow \infty .
$$

Our proof of Theorem 1.6 uses a compactness argument based on translations of the solution, combined with two crucial classification or Liouville type results for monostable equations in all space and in a half-space.

Theorem 1.6 will be used to control some of the integrals appearing in the proof of instability in $\mathbb{R}^{6}$. In such proof we will establish that the maximal saddle solution $\bar{u}$ is unstable in dimension 6 . The existence of such a maximal saddle solution, its monotonicity properties, as well as the existence of a minimal saddle solution $\underline{u}$ are the object of our second result. 
Theorem 1.7. For every nonlinearity $f$ satisfying conditions (1.9), there exist two saddle solutions $\underline{u}$ and $\bar{u}$ of $-\Delta u=f(u)$ in $\mathbb{R}^{2 m}$ which are minimal and maximal, respectively, in $\mathcal{O}=\{s>t\}$ in the following sense. For every solution $u$ of $-\Delta u=f(u)$ in $\mathbb{R}^{2 m}$ vanishing on the Simons cone and such that $u$ has the same sign as $s-t$, we have

$$
0<\underline{u} \leq u \leq \bar{u} \text { in } \mathcal{O} .
$$

As a consequence, we also have

$$
|\underline{u}| \leq|u| \leq|\bar{u}| \quad \text { in } \mathbb{R}^{2 m} .
$$

In addition, the maximal solution $\bar{u}$ satisfies:

(a) $-\partial_{t} \bar{u} \geq 0$ in $\mathbb{R}^{2 m}$. Furthermore, $-\partial_{t} \bar{u}>0$ in $\mathbb{R}^{2 m} \backslash\{t=0\}$ and $-\partial_{t} \bar{u}=0$ in $\{t=0\}$.

(b) $\partial_{s} \bar{u} \geq 0$ in $\mathbb{R}^{2 m}$. Furthermore, $\partial_{s} \bar{u}>0$ in $\mathbb{R}^{2 m} \backslash\{s=0\}$ and $\partial_{s} \bar{u}=0$ in $\{s=0\}$.

(c) As a consequence, $\partial_{z} \bar{u}>0$ in $\mathbb{R}^{2 m} \backslash\{0\}$; recall that $z=(s-t) / \sqrt{2}$.

(d) $\partial_{y} \bar{u}>0$ in $\mathcal{O}=\{s>t\}$; recall that $y=(s+t) / \sqrt{2}$. As a consequence, for every direction $\partial_{\eta}=\alpha \partial_{y}-\beta \partial_{t}$ with $\alpha$ and $\beta$ nonnegative constants, $\partial_{\eta} \bar{u}>0$ in $\{s>t>0\}$.

It is still an open problem to know if $\underline{u}=\bar{u}$ in dimensions $2 m \geq 4$, which would be equivalent to the uniqueness of saddle solution. This is only known to hold in dimension $2 m=2$ by a result of [16].

The cone of directions of monotonicity in $\mathcal{O}$ described in part $(\mathrm{d})$ of the theorem is optimal. Indeed, the level sets of a saddle solution (see Figure 2) intersect $\{t=0\}$ orthogonally by regularity of the solution as a function of the radial variables $s$ and $t$-i.e., fixed $s$, we must have $u_{t}=0$ at $\{t=0\}$ since $u(s, \cdot)$ is a $C^{1}$ radial function. On the other hand, by Theorem 1.6 on the asymptotic behavior of saddle solutions, the level sets at infinity become parallel to the Simons cone at a fixed distance (see also Figure 21). Thus, the cone of monotonicity in the theorem and figure is optimal.

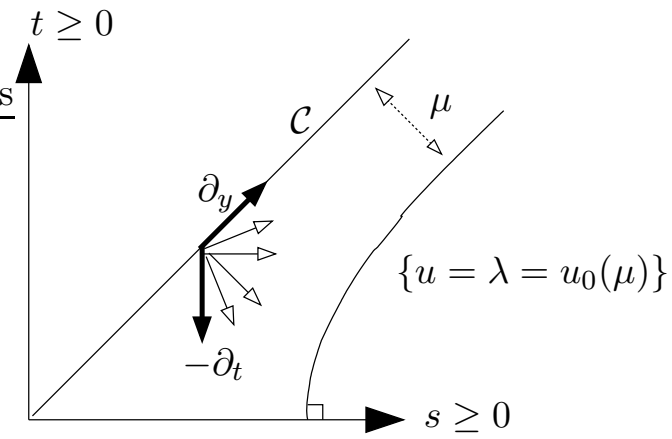

FiguRE 2. 


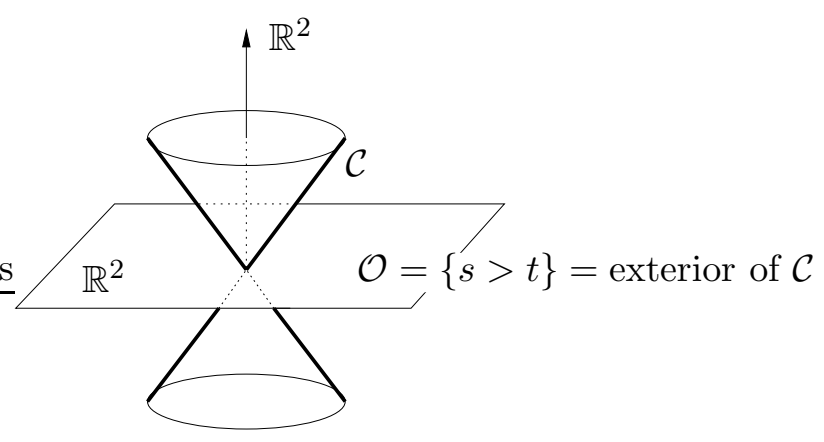

FiguRE 3.

We emphasize that our monotonicity results have been achieved without using the usual techniques of sliding or moving planes methods. The application of such methods to saddle solutions fails in $\mathbb{R}^{2 m}$ (for $2 m>2$ ) due to the particular geometry of saddle solutions in dimensions $2 m>2$. Indeed, after any translation in $\mathbb{R}^{2 m}$, the exterior $\mathcal{O}$ of the Simons cone does not contain (neither is contained) in the exterior of the translated cone; see Figure 3. This fact prevents the use of the sliding or moving planes methods. On the other hand, one could think of using these methods in the simple geometry of $\mathbb{R}^{2}$ for equation (1.12) in the $s$ and $t$ variables. But then, for $m>1$, the first order terms in the equation have the wrong monotonicity to apply any of the two methods. As we will see, our monotonicity results originates from the stability of saddle solutions within the region $\mathcal{O}$ - that is, under perturbations with compact support in $\mathcal{O}$.

To state our main theorem in dimension 6 , let us recall the notion of Morse index.

Definition 1.8. Let $f \in C^{1}(\mathbb{R})$. We say that a bounded solution $u$ of (1.1) has finite Morse index equal to $k \in\{0,1,2, \ldots\}$ if $k$ is the maximal dimension of a subspace $X_{k}$ of $C_{c}^{1}\left(\mathbb{R}^{n}\right)$ such that $Q_{u}(\xi)<0$ for every $\xi \in X_{k} \backslash\{0\}$. Here $C_{c}^{1}\left(\mathbb{R}^{n}\right)$ is the space of $C^{1}\left(\mathbb{R}^{n}\right)$ functions with compact support and $Q_{u}$ is defined in (1.10). If there is no such finite integer $k$, we then say that $u$ has infinite Morse index.

Every stable solution has finite Morse index equal to 0. It is also easy to verify that every solution with finite Morse index is stable outside of a compact set (see Theorem 1.9 and its proof in section 5 below for more details).

The following is our main result. It establishes that saddle solutions in dimension 6 are unstable outside of every compact set, and thus have infinite Morse index. It is the analogue of our four dimensional result in [14. Note that the result applies to a class of solutions which do not necessarily depend only on $s$ and $t$-and thus perhaps larger than the class of saddle solutions. 
Theorem 1.9. Let $f$ satisfy conditions (1.9). Then, every bounded solution of $-\Delta u=f(u)$ in $\mathbb{R}^{6}$ such that $u=0$ on the Simons cone $\mathcal{C}=\{s=t\}$ and $u$ is positive in $\mathcal{O}=\{s>t\}$ is unstable. Furthermore, every such solution $u$ is unstable outside of every compact set. That is, for every compact set $K$ of $\mathbb{R}^{6}$ there exists $\xi \in C^{1}\left(\mathbb{R}^{6}\right)$ with compact support in $\mathbb{R}^{6} \backslash K$ for which $Q_{u}(\xi)<0$, where $Q_{u}$ is defined in (1.10). As a consequence, $u$ has infinite Morse index in the sense of Definition 1.8.

In particular, every saddle solution as in Definition 1.3 is unstable outside of every compact set if $2 m=6$.

To establish this result, we prove that $\bar{u}$ is unstable outside of every compact set by constructing test functions

$$
\xi(y, z)=\eta(y) \bar{u}_{z}(y, z)
$$

such that $Q_{\bar{u}}(\xi)<0$. We need to use the asymptotic convergence and monotonicity results for $\bar{u}$ of Theorems 1.6 and 1.7. Since $\bar{u}$ is maximal, its instability outside of compact sets implies that this same instability property holds for all bounded solutions $u$ vanishing on the Simons cone $\mathcal{C}$ and positive in $\mathcal{O}$.

Let us comment on the Morse index of stationary surfaces, i.e., surfaces of zero mean curvature. The usual proof of the instability of the Simons cone in dimension 4 and 6 (see [23]) also leads to its instability outside of every compact set, and hence to its infinite Morse index property. A precise study of the Morse index of stationary surfaces close to the Simons cone is made in 3 through the analysis of intersection numbers. Note that in dimension 2, outside of a ball centered at the origin the Simons cone consists of 4 disconnected half-lines - a stable configuration. Note the analogy of these results with those for saddle solutions: we have proved that they have infinite Morse index in dimensions 4 and 6, while Schatzman established that in $\mathbb{R}^{2}$ the saddle solution has Morse index 1 .

The instability of saddle solutions in low dimensions is related to perturbations which do not vanish on the Simons cone, and hence, which change the zero level set of the solution. Indeed, the following result states that in all dimensions, every solution that vanishes on the Simons cone $\mathcal{C}$ and has the same sign as $s-t$ is stable under perturbations with compact support which vanish on the Simons cone.

Proposition 1.10. Let $f$ satisfy conditions (1.9). Then, every bounded solution $u$ of $-\Delta u=f(u)$ in $\mathbb{R}^{2 m}$ that vanishes on the Simons cone $\mathcal{C}=$ $\{s=t\}$ and has the same sign as $s-t$, is stable in $\mathbb{R}^{2 m}$ with respect to perturbations vanishing on $\mathcal{C}$. That is, $Q_{u}(\xi) \geq 0$ for all $\xi \in C^{1}\left(\mathbb{R}^{2 m}\right)$ with compact support and such that $\xi \equiv 0$ in $\mathcal{C}$, where $Q_{u}$ is defined in (1.10).

Remark 1.11. The last section shows that the maximal solution $\bar{u}$ is in some sense asymptotically stable at infinity in dimensions $2 m \geq 8$. This indicates that saddle-shaped solutions might be stable in dimensions $2 m \geq 8$. 
The recent work by Alessio, Calamai, and Montecchiari [4] concerns scalar saddle type solutions in $\mathbb{R}^{2}$ changing sign on more nodal lines than $x_{1}=$ $\pm x_{2}$, whereas Alama, Bronsard, and Gui in [1] studied vector-valued saddle solutions in $\mathbb{R}^{2}$.

The paper is organized as follows. In section 2 we present some of the results mentioned in this introductions and used later in the paper. Section 3 concerns the maximal and minimal saddle solutions and their properties; we prove Theorem 1.7 and Proposition 1.10. Section 4 is devoted to the study of the asymptotic behavior of saddle shaped solutions; we establish Theorem 1.6. Section 5 contains the proof of instability of saddle solutions in $\mathbb{R}^{6}$, Theorem 1.9. Finally, in section 6 we show that the maximal saddle solution $\bar{u}$ is in some sense asymptotically stable at infinity in dimensions $2 m \geq 8$.

\section{Preliminaries}

This section contains a more detailed description and statement of some of the results mentioned in the previous section. Most of these results will be used throughout the paper.

We begin with the existence of a solution in dimension one. The proof of this lemma, which follows from integrating the ODE $\ddot{u}-G^{\prime}(u)=0$, can be found in [5] - see also a sketch of the proof below, after the statement.

Lemma 2.1 (see e.g. [5]). Let $G \in C^{2}(\mathbb{R})$. There exists a bounded function $u_{0} \in C^{2}(\mathbb{R})$ satisfying

$$
\ddot{u}_{0}-G^{\prime}\left(u_{0}\right)=0 \quad \text { and } \quad \dot{u}_{0}>0 \quad \text { in } \mathbb{R}
$$

if and only if there exist two real numbers $m_{1}<m_{2}$ for which $G$ satisfies

$$
\begin{gathered}
G^{\prime}\left(m_{1}\right)=G^{\prime}\left(m_{2}\right)=0 \quad \text { and } \\
G>G\left(m_{1}\right)=G\left(m_{2}\right) \quad \text { in }\left(m_{1}, m_{2}\right) .
\end{gathered}
$$

In such case we have $m_{1}=\lim _{\tau \rightarrow-\infty} u_{0}(\tau)$ and $m_{2}=\lim _{\tau \rightarrow+\infty} u_{0}(\tau)$. Moreover, the solution $u_{0}=u_{0}(\tau)$ is unique up to translations of the independent variable $\tau$.

Adding a constant to $G$, assume that

$$
G\left(m_{1}\right)=G\left(m_{2}\right)=0 .
$$

Then, we have that

$$
\frac{\dot{u}_{0}^{2}}{2}=G\left(u_{0}\right) \quad \text { in } \mathbb{R}
$$

If in addition $G^{\prime \prime}\left(m_{1}\right) \neq 0$ and $G^{\prime \prime}\left(m_{2}\right) \neq 0$, then

$$
0<\dot{u}_{0}(\tau) \leq C e^{-c|\tau|} \quad \text { in } \mathbb{R}
$$

for some positive constants $C$ and $c$, and

$$
\int_{-\infty}^{+\infty}\left\{\frac{1}{2} \dot{u}_{0}(\tau)^{2}+G\left(u_{0}(\tau)\right)\right\} d \tau<+\infty .
$$


Given G satisfying (2.1), (2.2), and (2.3), to construct $u_{0}$ we simply choose any $m_{0} \in\left(m_{1}, m_{2}\right)$ and define

$$
\phi(\sigma)=\int_{m_{0}}^{\sigma} \frac{d w}{\sqrt{2(G(w))}} \quad \text { for } \sigma \in\left(m_{1}, m_{2}\right) .
$$

Then let $u_{0}:=\phi^{-1}$ be the inverse function of $\phi$. This formula is found multiplying $\ddot{u}-G^{\prime}(u)=0$ by $\dot{u}$ and integrating the equation - which also gives the necessity of conditions (2.1) and (2.2) for existence. The above definition of $u_{0}$ leads automatically to (2.4). Under the additional hypothesis $G^{\prime \prime}\left(m_{i}\right) \neq 0, G$ behaves like a quadratic function near each $m_{i}$. Using the expression above for $\phi$, this gives that $\phi$ blows-up logarithmically at $m_{i}$, and thus its inverse function $u_{0}$ attains its limits $m_{i}$ at $\pm \infty$ exponentially. From this and identity (2.4), the exponential decay (2.5) for $\dot{u}_{0}$, as well as (2.6), follow.

In relation with assumptions (2.1) and (2.2) on $G$, our hypothesis

$$
G \geq 0=G( \pm M) \text { in } \mathbb{R} \text { and } G>0 \text { in }(-M, M)
$$

appearing in (1.9), guarantees the existence of an increasing solution $u_{0}$ of (1.1) in dimension 1 , that is in all of $\mathbb{R}$, taking values onto $(-M, M)$, as stated in Lemma 2.1. The nonlinearities $f$ that satisfy property (2.7) are of "balanced bistable type", while the potentials $G$ are of "double-well type". Typical examples are the Allen-Cahn (or scalar Ginzburg-Landau) equation $-\Delta u=u-u^{3}$, where $G(u)=(1 / 4)\left(1-u^{2}\right)^{2}$ and $M=1$, and also the equation $-\Delta u=\sin (\pi u)$, for which $M=1$ and $G(u)=(1 / \pi)(1+\cos (\pi u))$.

Since the increasing solution whose existence is established above is unique up to translations of the independent variable, we normalize it to vanish at the origin and we call it $u_{0}$. Thus, we have

$$
\left\{\begin{array}{l}
u_{0}: \mathbb{R} \rightarrow(-M, M) \\
u_{0}(0)=0, \dot{u}_{0}>0 \text { in } \mathbb{R}, \text { and } \\
-\ddot{u}_{0}=f\left(u_{0}\right) \text { in } \mathbb{R} .
\end{array}\right.
$$

For the Allen-Cahn nonlinearity $f(u)=u-u^{3}$, the solution $u_{0}$ can be computed explicitly and it is given by $u_{0}(\tau)=\tanh (\tau / \sqrt{2})$.

We can now consider the family of $1 \mathrm{D}$ solutions to (1.1) in $\mathbb{R}^{n}$, given by

$$
u_{b, c}(x)=u_{0}(b \cdot x+c) \quad \text { for } x \in \mathbb{R}^{n},
$$

for every given $b \in \mathbb{R}^{n}$ with $|b|=1$ and $c \in \mathbb{R}$. Under hypothesis (2.7) on the nonlinearity, every $1 \mathrm{D}$ solution $u_{b, c}$ is a global minimizer of (1.1), by Theorem 1.1 in the introduction when $f(u)=u-u^{3}$ (or by the more general result in [2]). In particular, $u_{b, c}$ is a stable solution. By Savin's result, Theorem 1.2 above, we know that $1 \mathrm{D}$ solutions are the only global minimizers of the Allen-Cahn equation (1.2) if $n \leq 7$.

In $\mathbb{R}^{8}$ it is expected that global minimizers which are not $1 \mathrm{D}$ exist. As argued in the introduction, natural candidates to be minimizers of this type are saddle-shaped solutions. 
In [14] we established the existence of saddle solutions to (1.1) (see Theorem 1.4 in the Introduction). By classical elliptic regularity theory, it is well known that for $f \in C^{1}(\mathbb{R})$, every bounded solution of $-\Delta u=f(u)$ in $\mathbb{R}^{n}$ satisfies $u \in C^{2, \alpha}\left(\mathbb{R}^{n}\right)$ for all $0<\alpha<1$, and thus it is a classical solution. In particular, saddle solutions are classical solutions.

Moreover (see [14]), there exists a saddle solution $u$ satisfying $|u|<M$ in $\mathbb{R}^{2 m}$, as well as the energy estimate

$$
\mathcal{E}\left(u, B_{R}\right)=\int_{B_{R}}\left\{\frac{1}{2}|\nabla u|^{2}+G(u)\right\} d x \leq C R^{2 m-1} \quad \text { for all } R>1,
$$

where $C$ is a constant independent of $R$ and $B_{R}$ denotes the open ball of radius $R$ centered at 0 . Both these estimates as well as existence itself are establish assuming only the first two conditions on $f$ in (1.9).

A crucial ingredient that we used in the proof of the instability theorem in dimension 4 and which we will use also in this paper is the following pointwise estimate.

Proposition 2.2 ([14]). Let $f \in C^{1}(\mathbb{R})$ satisfy (1.9). If $u$ is a bounded solution of $-\Delta u=f(u)$ in $\mathbb{R}^{2 m}$ that vanishes on the Simons cone $\mathcal{C}=\{s=$ $t\}$, then

$$
|u(x)| \leq\left|u_{0}(\operatorname{dist}(x, \mathcal{C}))\right|=\left|u_{0}\left(\frac{s-t}{\sqrt{2}}\right)\right| \quad \text { for all } x \in \mathbb{R}^{2 m},
$$

where $u_{0}$ is defined by (2.8) and $\operatorname{dist}(\cdot, \mathcal{C})$ denotes the distance to the Simons cone, which is equal to $|s-t| / \sqrt{2}$.

In addition, the function $u_{0}((s-t) / \sqrt{2})$ is a supersolution of $-\Delta u=f(u)$ in the set $\mathcal{O}=\{s>t\}$.

Estimate (2.11) follows easily from an important estimate for global solutions of semilinear equations, called Modica estimate [25].

Finally we recall here the statement of the theorem of Jerison and Monneau 24] establishing that the existence of a bounded, even with respect to each coordinate, global minimizer of $(1.2)$ in $\mathbb{R}^{n-1}$ would yield the existence of a counter-example to the conjecture of De Giorgi in $\mathbb{R}^{n}$.

Theorem 2.3 (Jerison-Monneau [24]). Let $G \in C^{2}(\mathbb{R})$ satisfy

$$
G \geq 0 \text { in } \mathbb{R}, \quad G>0 \text { in }(-1,1) \text {, and } G(1)=G(-1)=0 .
$$

Assume that there exists a global minimizer $v$ of $\Delta v=G^{\prime}(v)$ on $\mathbb{R}^{n-1}$ such that $|v|<1$ and $v$ is even with respect to each coordinate $x_{i}, 1 \leq i \leq n-1$. Then, for each $\gamma \in(0, \sqrt{2 G(v(0))})$ there exists a solution $u \in C^{2}\left(\mathbb{R}^{n}\right)$ to

$$
\Delta u=G^{\prime}(u) \quad \text { in } \mathbb{R}^{n}
$$

satisfying

$$
|u| \leq 1 \quad \text { and } \quad \partial_{x_{n}} u>0 \quad \text { in } \mathbb{R}^{n}
$$

and such that, for one $\lambda \in \mathbb{R}$, the set $\{u=\lambda\}$ is not a hyperplane. 
Moreover, this solution $u$ is a global minimizer in $\mathbb{R}^{n}$, it is even in the first $n-1$ coordinates, and satisfies $\partial_{x_{n}} u(0)=\gamma$ and $u(0)=v(0)$.

\section{Minimal and maximal Saddle Solutions}

As in the work of Dang, Fife, and Peletier [16] in dimension 2, in this section we show the existence in higher dimensions of a minimal, $\underline{u}$, and a maximal, $\bar{u}$, saddle solutions and we prove the monotonicity properties of $\bar{u}$ stated in Theorem 1.7. We recall that we establish, among other properties, that the maximal solution is increasing in the $z$ direction, fact that we will use in the proof of instability of saddle solutions in dimension 6 (see section 5 ).

Moreover, we prove Proposition 1.10 stating the stability under perturbations vanishing on the Simons cone of saddle solutions.

The proof of Theorem 1.7 and of Proposition 1.10 are given at the end of subsection 3.2 .

3.1. Existence of minimal and maximal solutions. For $R>0$, consider the open sets

$$
T_{R}=\left\{x \in \mathbb{R}^{2 m}: t<s<R\right\}
$$

and

$$
O_{R}=\mathcal{O} \cap B_{R}=\left\{x \in \mathbb{R}^{2 m}: s>t\right\} \cap B_{R},
$$

where $B_{R}$ is the open ball in $\mathbb{R}^{2 m}$ of radius $R$ centered at 0 . Note that $T_{R} \supset \mathcal{O}_{R}$.

In the next lemmas, we will need the following simple facts on the nonlinearity.

Remark 3.1. Let $f$ satisfy assumptions (1.9). Define

$$
g(\rho):=f(\rho)-f^{\prime}(M) \rho \quad \text { for } 0 \leq \rho \leq M .
$$

Then, $g$ is positive and increasing in $(0, M)$. Indeed, since $f^{\prime}$ is decreasing in $(0, M), g^{\prime}(\rho)=f^{\prime}(\rho)-f^{\prime}(M)>0$ in $(0, M)$. That is, $g$ is increasing in $(0, M)$. Since $g(0)=0$, we deduce that $g>0$ in $(0, M)$.

Another fact that we will use is that $f(\rho) / \rho$ is decreasing in $(0, M)$. Indeed, given $0<\rho<M$ there exists $\rho_{1}$ with $0<\rho_{1}<\rho$ and

$$
\frac{f(\rho)}{\rho}=\frac{f(\rho)-f(0)}{\rho-0}=f^{\prime}\left(\rho_{1}\right)>f^{\prime}(\rho)
$$

since $f^{\prime}$ is decreasing in $(0, M)$. Therefore,

$$
\left(\frac{f(\rho)}{\rho}\right)^{\prime}=\frac{f^{\prime}(\rho) \rho-f(\rho)}{\rho^{2}}=\frac{f^{\prime}(\rho)-f^{\prime}\left(\rho_{1}\right)}{\rho}<0 .
$$

The following definition and remark on stability of positive solutions will be used in some of the next lemmas, as well as in the proof of Proposition 1.10 . 
Definition 3.2. Let $\Omega \subset \mathbb{R}^{n}$ be an open set. We say that a bounded solution $u$ of $-\Delta u=f(u)$ in $\Omega$ is semi-stable in $\Omega$ if the second variation of energy $\delta^{2} \mathcal{E} / \delta^{2} \xi$ with respect to perturbations $\xi \in H_{0}^{1}(\Omega)$ is nonnegative. That is, if

$$
Q_{u}(\xi):=\int_{\Omega}\left\{|\nabla \xi|^{2}-f^{\prime}(u) \xi^{2}\right\} d x \geq 0 \quad \text { for all } \xi \in H_{0}^{1}(\Omega) .
$$

Remark 3.3. Let $f$ satisfy assumptions (1.9) and $\Omega \subset \mathbb{R}^{n}$ be an open set (bounded or unbounded). Let $u$ be a positive solution of $-\Delta u=f(u)$ in $\Omega$ such that $0<u<M$ in $\Omega$. Then, $u$ is a semi-stable solution in $\Omega$.

The proof of this fact is simple. By (3.2), $f^{\prime}(w) \leq f(w) / w$ for all real numbers $w \in(0, M)$. Hence we have

$$
-\Delta u=f(u) \geq f^{\prime}(u) u \quad \text { in } \Omega .
$$

That is, $u$ is a positive supersolution of the linearized problem $-\Delta-f^{\prime}(u)$ at $u$ in all of $\Omega$. We claim that, as a consequence, the quadratic form $Q_{u}(\xi)$ is nonnegative for all $\xi \in C^{1}$ with compact support in $\Omega$. By approximation, the same holds for all $\xi \in H_{0}^{1}(\Omega)$.

This claim can be proved in two different ways. First, by a simple integration by parts argument. It consists of taking any $\xi \in C^{1}$ with compact support in $\Omega$, multiplying (3.4) by $\xi^{2} / u$, and integrating by parts to get

$$
\int_{\Omega} f^{\prime}(u) \xi^{2}=\int_{\Omega} f^{\prime}(u) u \frac{\xi^{2}}{u} \leq \int_{\Omega}(-\Delta u) \frac{\xi^{2}}{u} \leq \int_{\Omega} \nabla u \nabla \xi \frac{2 \xi}{u}-\int_{\Omega} \frac{|\nabla u|^{2}}{u^{2}} \xi^{2} .
$$

Now, using the Cauchy-Schwarz inequality, we are led to (3.3).

Another proof of the claim is the following. Since the linearized operator has a positive supersolution, its principal eigenvalue is nonnegative (see [12]). Since the principal eigenvalue coincides with the first eigenvalue, Rayleigh criterion gives (3.3).

Our first lemma concerns the existence of a maximal solution in $T_{R}$ and some of its properties.

Lemma 3.4. Let $f$ satisfy conditions (1.9). Then, there exists a positive solution $\bar{u}_{R}$ of

$$
\left\{\begin{aligned}
-\Delta \bar{u}_{R}=f\left(\bar{u}_{R}\right) & \text { in } T_{R}:=\{t<s<R\} \\
\bar{u}_{R}=u_{0}(z) & \text { on } \partial T_{R},
\end{aligned}\right.
$$

with $0<\bar{u}_{R}<M$ in $T_{R}$, which is maximal in the following sense. We have that $\bar{u}_{R} \geq u$ in $T_{R}$ for every positive solution $u$ of $-\Delta u=f(u)$ in $T_{R}$ satisfying $u \leq u_{0}(z)$ in all of $\overline{T_{R}}$.

Moreover, $\bar{u}_{R}$ is semi-stable in $T_{R}$ in the sense of Definition 3.2. and $\bar{u}_{R}$ depends only on $s$ and $t$.

Proof. Let $u_{0}$ be the solution in $\mathbb{R}$ defined by $(2.8)$ and $z=(s-t) / \sqrt{2}$. With $g$ as defined in (3.1), we write equation $-\Delta u=f(u)$ as $\left\{-\Delta-f^{\prime}(M)\right\} u=$ $g(u)$. 
We use the method of monotone iteration. Define a sequence of functions $\bar{u}_{R, k}$ by $\bar{u}_{R, 0}(x)=u_{0}(z)$ and by solving the linear problems

$$
\left\{\begin{aligned}
\left\{-\Delta-f^{\prime}(M)\right\} \bar{u}_{R, k+1} & =g\left(\bar{u}_{R, k}\right) & & \text { in } T_{R} \\
\bar{u}_{R, k} & =u_{0}(z) & & \text { on } \partial T_{R} .
\end{aligned}\right.
$$

Since $-\Delta-f^{\prime}(M)$ is obtained by adding a positive constant to $-\Delta$, it satisfies the maximum principle and hence the above problem admits a unique solution $\bar{u}_{R, k+1}=\bar{u}_{R, k+1}(x)$. Furthermore (and here we argue by induction), since the problem and its data are invariant by orthogonal transformations in the first (respectively, in the last) $m$ variables $x_{i}$, the solution $\bar{u}_{R, k+1}$ depends only on $s$ and $t$.

Using that $\bar{u}_{R, 0}(x)=u_{0}(z)$ is a supersolution of (3.6) (as stated in Proposition 2.2), let us show that the sequence $\bar{u}_{R, k}$ is nonincreasing in $k$. More precisely,

$$
u_{0}(z)=\bar{u}_{R, 0} \geq \bar{u}_{R, 1} \geq \cdots \geq \bar{u}_{R, k} \geq \bar{u}_{R, k+1} \geq \cdots \geq 0 \quad \text { in } T_{R} .
$$

Indeed, we have

$$
\left\{-\Delta-f^{\prime}(M)\right\} \bar{u}_{R, 1}=f\left(\bar{u}_{R, 0}\right)-f^{\prime}(M) \bar{u}_{R, 0} \leq\left\{-\Delta-f^{\prime}(M)\right\} \bar{u}_{R, 0}
$$

in $T_{R}$. Hence $\bar{u}_{R, 1} \leq \bar{u}_{R, 0}$. We use now that 0 a subsolution of (3.6). Since $\left\{-\Delta-f^{\prime}(M)\right\} 0=0 \leq g\left(\bar{u}_{R, 0}\right)$ in $T_{R}$ and $0 \leq u_{0}(z)$ on $\partial T_{R}$, we deduce $\bar{u}_{R, 1} \geq 0$.

Assume now that $0 \leq \bar{u}_{R, k} \leq \bar{u}_{R, k-1}$ in $T_{R}$, for some $k \geq 1$. Then, since $g$ is increasing in $(0, M)$ (see Remark 3.1),$g\left(\bar{u}_{R, k}\right) \leq g\left(\bar{u}_{R, k-1}\right)$, and thus

$$
\left\{-\Delta-f^{\prime}(M)\right\} \bar{u}_{R, k+1}=g\left(\bar{u}_{R, k}\right) \leq g\left(\bar{u}_{R, k-1}\right)=\left\{-\Delta-f^{\prime}(M)\right\} \bar{u}_{R, k} .
$$

Again by the maximum principle $\bar{u}_{R, k+1} \leq \bar{u}_{R, k}$. Besides, $\bar{u}_{R, k+1} \geq 0$ since $g\left(\bar{u}_{R, k}\right) \geq 0$.

Next, by monotone convergence this sequence converges to a nonnegative solution $\bar{u}_{R}$ of $-\Delta u=f(u)$ in $T_{R}$, which depends only on $s$ and $t$, and such that $\bar{u}_{R} \equiv 0$ on $\mathcal{C} \cap \overline{T_{R}}$. Since $\bar{u}_{R}=u_{0}(z)$ on $\{s=R\} \cap \overline{T_{R}}, \bar{u}_{R}$ is not identically 0 , and thus the strong maximum principle and $f(0)=0$ lead to $\bar{u}_{R}>0$ in $T_{R}$.

Moreover, $\bar{u}_{R}$ is maximal as stated in the lemma. Indeed, by assumption $0<u \leq u_{0}(z)$ in all $T_{R}$. Assume now that $0<u \leq \bar{u}_{R, k}$ for some $k \geq 0$. We then have

$$
\left\{-\Delta-f^{\prime}(M)\right\} u=g(u) \leq g\left(\bar{u}_{R, k}\right)=\left\{-\Delta-f^{\prime}(M)\right\} \bar{u}_{R, k+1}
$$

in $T_{R}$ and $u \leq u_{0}(z)=\bar{u}_{R, k+1}$ on $\partial T_{R}$. Thus $u \leq \bar{u}_{R, k+1}$ in $T_{R}$. By induction $u \leq \bar{u}_{R, k+1}$ for all $k$ and hence $u \leq \bar{u}_{R}$ in $T_{R}$.

Finally, the semi-stability of $\bar{u}_{R}$ in $T_{R}$ follows directly from Remark 3.3 , 
Proposition 3.5. Let $f$ satisfy conditions (1.9). Then, there exists a positive solution $\bar{u}$ of

$$
\left\{\begin{aligned}
-\Delta \bar{u} & =f(\bar{u}) & & \text { in } \mathcal{O}=\{s>t\} \\
\bar{u} & =0 & & \text { on } \mathcal{C}=\partial \mathcal{O}
\end{aligned}\right.
$$

with $0<\bar{u}<M$ in $\mathcal{O}$, which is maximal in the following sense. We have that $\bar{u} \geq u$ in $\mathcal{O}$ for every bounded solution $u$ of $-\Delta u=f(u)$ in $\mathbb{R}^{2 m}$ that vanishes on the Simons cone and has the same sign as $s-t$.

In addition, $\bar{u}$ depends only on $s$ and $t$.

Proof. By elliptic estimates and a compactness argument, the limit as $R \rightarrow$ $\infty$ of the solutions $\bar{u}_{R}$ of Lemma 3.4 exists (up to subsequences) in every compact set of $\overline{\mathcal{O}}$. We obtain a solution $\bar{u}$ in $\mathcal{O}=\{s>t\}$ such that $\bar{u}=0$ on $\mathcal{C}$ and $0 \leq \bar{u} \leq M$ in $\mathcal{O}$. Clearly, $u$ depends only on $s$ and $t$.

Let us next establish the maximality of $\bar{u}$. Let $u$ be a solution as in the statement. By Proposition 2.2, we have $u \leq u_{0}(z)$ in $\mathcal{O}$. Thus, by Lemma 3.4, $u \leq \bar{u}_{R}$ in $T_{R}$ for all $R$. It follows that $u \leq \bar{u}$ in $\mathcal{O}$.

Finally, we show that $\bar{u}$ is not identically 0 . Indeed, by maximality of $\bar{u}$ and the existence of saddle solution $u$ of Theorem 1.4, we deduce that $\bar{u} \geq u>0$ in $\mathcal{O}$. An alternative way to prove $u \not \equiv 0$ is to use the subsolution of next remark, by placing such subsolution below all maximal solutions $\bar{u}_{R}$.

Remark 3.6. The following subsolution is useful in several arguments. Let $R$ be large enough such that $\mathcal{O}_{R}=\mathcal{O} \cap B_{R}$ contains a closed ball $B$ of sufficiently large radius to guarantee that $\lambda_{1}<f^{\prime}(0)$, where $\lambda_{1}$ is the first Dirichlet eigenvalue of the Laplacian in $B$. Let $\phi_{1}>0$ in $B$ be the first Dirichlet eigenfunction of $-\Delta$ in $B$ and $\varepsilon>0$ a constant small enough. Then,

$$
-\Delta\left(\varepsilon \phi_{1}\right)=\lambda_{1} \varepsilon \phi_{1} \leq f\left(\varepsilon \phi_{1}\right) \text { in } B .
$$

The last inequality holds since $f\left(\varepsilon \phi_{1}\right) /\left(\varepsilon \phi_{1}\right) \geq f^{\prime}\left(\varepsilon \phi_{1}\right)$ for $\varepsilon$ small (recall that $f$ is concave in $(0, M))$, and also $f^{\prime}\left(\varepsilon \phi_{1}\right)>\lambda_{1}$ for $\varepsilon$ small, since we chose $B$ such that $f^{\prime}(0)>\lambda_{1}$. Therefore, $\varepsilon \phi_{1}$ extended by zero in $\mathcal{O}_{R} \backslash B$ is a subsolution to problem

$$
\left\{\begin{aligned}
-\Delta v & =f(v) & & \text { in } \mathcal{O}_{R} \\
v & =0 & & \text { on } \partial \mathcal{O}_{R}
\end{aligned}\right.
$$

which is positive in $B$, a ball with compact closure contained in $\mathcal{O}_{R}$.

Next we start studying the existence of a minimal saddle solution. Among other things, the next lemma establishes the uniqueness of positive solution in $\mathcal{O}_{R}$ taking values in $(0, M)$ and vanishing on $\partial \mathcal{O}_{R}$. This uniqueness is a well known general result that only requires $f(\rho) / \rho$ to be decreasing in $(0, M)$. 
Lemma 3.7. Let $f$ satisfy conditions (1.9). Then, for $R$ large enough, there exists a unique positive solution $\underline{u}_{R}$ of

$$
\left\{\begin{aligned}
-\Delta u & =f(u) & & \text { in } \mathcal{O}_{R}:=\{s>t\} \cap B_{R} \\
u & <M & & \text { in } \mathcal{O}_{R} \\
u & =0 & & \text { on } \partial \mathcal{O}_{R},
\end{aligned}\right.
$$

which is minimal in the following sense. We have that $\underline{u}_{R} \leq u$ in $\mathcal{O}_{R}$ for every positive solution $u$ of $-\Delta u=f(u)$ in $\mathcal{O}_{R}$.

Moreover, $\underline{u}_{R}$ is semi-stable in $\mathcal{O}_{R}$ in the sense of Definition 3.2, and $\underline{u}_{R}$ depends only on $s$ and $t$.

Proof. We claim that, for $R$ large enough, there exists a maximal positive solution $\bar{v}$ of (3.8). This is proved by monotone iteration as in the proof of Lemma 3.4, now with zero boundary conditions on $\partial \mathcal{O}_{R}$, and starting the iteration with $\bar{v}_{R, 0} \equiv M$, a supersolution of (3.8). Here, we use the subsolution of Remark 3.6 to guarantee that the limit of the iteration, $\bar{v}$, is not identically zero, and thus positive.

Using this maximal solution, we can now prove the uniqueness statement of the lemma. Let $v$ be a positive solution of (3.8). Since $\bar{v}$ is maximal we have $v \leq \bar{v}$ in $\mathcal{O}_{R}$.

To avoid integrating by parts in all of $\mathcal{O}_{R}$ (since $\partial \mathcal{O}_{R}$ is not a Lipschitz domain at $0 \in \partial \mathcal{O}_{R}$ ), we consider a ball $B_{\varepsilon}$ centered at the origin and of radius $\varepsilon$. The integration by parts formula can now be used in the region $\mathcal{O}_{R} \backslash B_{\varepsilon}$. Multiplying the equation for $\bar{v}$ by $v$ and integrating over $\mathcal{O}_{R}$ we get

$$
\begin{aligned}
\int_{\mathcal{O}_{R}} f(\bar{v}) v & =\int_{\mathcal{O}_{R} \cap B_{\varepsilon}}(-\Delta \bar{v}) v+\int_{\mathcal{O}_{R} \backslash B_{\varepsilon}}(-\Delta \bar{v}) v \\
& =\int_{\mathcal{O}_{R} \cap B_{\varepsilon}}(-\Delta \bar{v}) v+\int_{\mathcal{O}_{R} \backslash B_{\varepsilon}} \nabla \bar{v} \nabla v-\int_{\partial B_{\varepsilon} \cap \mathcal{O}_{R}} v \nabla \bar{v} \cdot \nu
\end{aligned}
$$

where $\nu$ is the outward normal on $\partial B_{\varepsilon}$ to $\mathcal{O}_{R} \backslash B_{\varepsilon}$. Similarly, we multiply now the equation for $v$ by $\bar{v}$ and obtain

$$
\begin{aligned}
\int_{\mathcal{O}_{R}} f(v) \bar{v} & =\int_{\mathcal{O}_{R} \cap B_{\varepsilon}}(-\Delta v) \bar{v}+\int_{\mathcal{O}_{R} \backslash B_{\varepsilon}}(-\Delta v) \bar{v} \\
& =\int_{\mathcal{O}_{R} \cap B_{\varepsilon}}(-\Delta v) \bar{v}+\int_{\mathcal{O}_{R} \backslash B_{\varepsilon}} \nabla v \nabla \bar{v}-\int_{\partial B_{\varepsilon} \cap \mathcal{O}_{R}} \bar{v} \nabla v \cdot \nu .
\end{aligned}
$$

Subtracting, we obtain

$$
\begin{gathered}
\int_{\mathcal{O}_{R}}\left(\frac{f(\bar{v})}{\bar{v}}-\frac{f(v)}{v}\right) \bar{v} v=\int_{\mathcal{O}_{R} \cap B_{\varepsilon}}((\Delta v) \bar{v}-(\Delta \bar{v}) v)- \\
-\int_{\partial B_{\varepsilon} \cap \mathcal{O}_{R}}(v \nabla \bar{v} \cdot \nu-\bar{v} \nabla v \cdot \nu) .
\end{gathered}
$$

We claim that every positive solution $v$ of $(3.8)$ belongs to $C^{2}\left(\overline{\mathcal{O}_{R}}\right)$, that is, is $C^{2}$ up to the boundary. This is proved using the ideas of the proof 
in 14] of Theorem 1.4 above. Namely, we first do odd reflection of $v$ with respect to $\mathcal{C}$ to obtain a solution in $B_{R} \backslash\{0\}$. Then, by a standard capacity argument we see that $v$ is a solution in fact in all $B_{R}$. Then, classical elliptic theory gives $v \in C^{2, \alpha}\left(\overline{B_{R}}\right)$.

Thus, we can let $\varepsilon$ tend to zero above and obtain

$$
\int_{\mathcal{O}_{R}}\left(\frac{f(\bar{v})}{\bar{v}}-\frac{f(v)}{v}\right) \bar{v} v=0
$$

Since $0<v \leq \bar{v}<M$, Remark 3.1 leads to

$$
\frac{f(\bar{v})}{\bar{v}}-\frac{f(v)}{v} \leq 0 \quad \text { in } \mathcal{O}_{R} .
$$

Thus, the integrand in (3.9) is nonpositive. Its integral being zero leads to such integrand being identically zero, and thus $v \equiv \bar{v}$.

We have proved that, for $R$ large enough, there exists a unique positive solution of (3.8), that we denote by $\underline{u}_{R}$ as in the statement of the lemma. This solution agrees with the maximal solution $\bar{v}$ obtained by monotone iteration at the beginning of the proof. Thus, the solution depends only on $s$ and $t$. In addition, its semi-stability in $\mathcal{O}_{R}$ follows from Remark 3.3 .

It only remains to prove the statement on minimality. For this, let $u$ be positive and satisfy $-\Delta u=f(u)$ in $\mathcal{O}_{R}$. Choose $\varepsilon>0$ small enough such that the subsolution of Remark [3.6 is smaller than $\min (u, M)$ (a supersolution) in all of $\mathcal{O}_{R}$. Then, in between this subsolution and supersolution there is a solution of (3.8) which, by uniqueness, must coincide with $\underline{u}_{R}$. Thus $\underline{u}_{R} \leq \min (u, M) \leq u$ in $\mathcal{O}_{R}$ as claimed.

Proposition 3.8. Let $f$ satisfy conditions (1.9). Then, there exists a positive solution $\underline{u}$ of

$$
\left\{\begin{aligned}
-\Delta \underline{u} & =f(\underline{u}) & & \text { in } \mathcal{O} \\
\underline{u} & =0 & & \text { on } \mathcal{C}=\partial \mathcal{O},
\end{aligned}\right.
$$

which is minimal in the following sense. We have that $\underline{u} \leq u$ in $\mathcal{O}$ for every bounded solution $u$ of $-\Delta u=f(u)$ in $\mathbb{R}^{2 m}$ that vanishes on the Simons cone and has the same sign as $s-t$.

In addition, $\underline{u}$ depends only on $s$ and $t$.

Proof. Note that, by minimality, the solutions $\underline{u}_{R}$ of the previous lemma form an increasing sequence in $R$. Thus, letting $R \rightarrow \infty$ we obtain a positive solution $\underline{u}$, which depends only on $s$ and $t$. Finally, the statement on minimality of $\underline{\underline{u}}$ follows immediately from the one for $\underline{u}_{R}$ in Lemma 3.7

3.2. Monotonicity properties. We now prove monotonicity properties for the maximal solution $\bar{u}$ of Proposition 3.5 .

Lemma 3.9. The maximal solution $\bar{u}_{R}$ of Lemma 3.4 satisfies $\partial_{t} \bar{u}_{R} \leq 0$ in $T_{R}$. 
Proof. We will see that we can prove the monotonicity property with two different methods, one based on the semi-stability of $\bar{u}_{R}$, and the other on the maximality of $\bar{u}_{R}$ and thus the monotone iteration method to construct it. In any case, we first must check the right monotonicity on the boundary $\partial T_{R}$.

Since $\bar{u}_{R} \geq 0$ in $T_{R}$ and vanishes on $\{s=t \leq R\}$, we have that $\partial_{t} \bar{u}_{R} \leq 0$ on $\{s=t \leq R\}$. On the remaining part of $\partial T_{R}$, which is $\{t<s=R\}$, $\bar{u}_{R}(x)=u_{0}(z)=u_{0}((s-t) / \sqrt{2})$. Thus, for $t<s=R$,

$$
\partial_{t} \bar{u}_{R}(R, t)=-\frac{1}{\sqrt{2}} \dot{u}_{0}(z)<0 .
$$

Therefore $\partial_{t} \bar{u}_{R} \leq 0$ on $\partial T_{R}$.

It follows that the positive part $\left(\partial_{t} \bar{u}_{R}\right)^{+}$belongs to $H_{0}^{1}\left(T_{R}\right)$, and hence it is an admissible test function for the quadratic form $Q_{\bar{u}_{R}}$ in $T_{R}$. Recall that by Lemma 3.4, $\bar{u}_{R}$ is semi-stable in $T_{R}$, that is,

$$
Q_{\bar{u}_{R}}(\xi)=\int_{T_{R}}\left\{|\nabla \xi|^{2}-f^{\prime}\left(\bar{u}_{R}\right) \xi^{2}\right\} d x \geq 0,
$$

for all $\xi \in H_{0}^{1}\left(T_{R}\right)$. Now, since $\bar{u}_{R}$ is a solution, in coordinates $s$ and $t$ we have

$$
-\partial_{s s} \bar{u}_{R}-\partial_{t t} \bar{u}_{R}-(m-1) \frac{\partial_{s} \bar{u}_{R}}{s}-(m-1) \frac{\partial_{t} \bar{u}_{R}}{t}=f\left(\bar{u}_{R}\right) \text { in } T_{R} \cap\{t>0\} .
$$

Differentiating with respect to $t$ we get

$$
-\Delta \partial_{t} \bar{u}_{R}-f^{\prime}\left(\bar{u}_{R}\right) \partial_{t} \bar{u}_{R}=-\frac{m-1}{t^{2}} \partial_{t} \bar{u}_{R} \quad \text { in } T_{R} \cap\{t>0\} .
$$

Therefore, multiplying (3.11) by $\left(\partial_{t} \bar{u}_{R}\right)^{+}$and integrating by parts, we have that

$$
\begin{aligned}
0 & \leq Q_{\bar{u}_{R}}\left(\left(\partial_{t} \bar{u}_{R}\right)^{+}\right) \\
& =\int_{T_{R}}\left\{\left|\nabla\left(\partial_{t} \bar{u}_{R}\right)^{+}\right|^{2}-f^{\prime}\left(\bar{u}_{R}\right)\left(\left(\partial_{t} \bar{u}_{R}\right)^{+}\right)^{2}\right\} d x \\
& =-\int_{T_{R}} \frac{m-1}{t^{2}}\left(\left(\partial_{t} \bar{u}_{R}\right)^{+}\right)^{2} d x \leq 0 ;
\end{aligned}
$$

note that the set $\{t=0\}$ is of zero measure. Since the last integrand is nonnegative, its integral being zero leads to $\left(\partial_{t} \bar{u}_{R}\right)^{+} \equiv 0$ in $T_{R} \cap\{t>0\}$ and thus in $T_{R}$. This finishes the proof.

As mentioned in the beginning of the proof, $\partial_{t} \bar{u}_{R} \leq 0$ can also be established using the maximality of the solution. Indeed, by its maximality, $\bar{u}_{R}$ must be equal to the solution constructed by monotone iteration in (3.6). Assuming $\partial_{t} \bar{u}_{R, k} \leq 0$ (which clearly holds for $k=0$ ), we differentiate the equation in (3.6) to obtain

$$
\left\{-\Delta-f^{\prime}(M)+\frac{m-1}{t}\right\} \partial_{t} \bar{u}_{R, k+1}=g^{\prime}\left(\bar{u}_{R, k}\right) \partial_{t} \bar{u}_{R, k} \quad \text { in } T_{R} .
$$


The right hand side is nonpositive by inductive hypothesis. Since the operator $-\Delta-f^{\prime}(M)+(m-1) / t$ satisfies the maximum principle (due to the positive signs of the zeroth order coefficients), we deduce $\partial_{t} \bar{u}_{R, k+1} \leq 0$.

In a similar way we now establish a sign for $\partial_{y} \bar{u}_{R}$ in $T_{R}$.

Lemma 3.10. The maximal solution $\bar{u}_{R}$ of Lemma 3.4 satisfies $\partial_{y} \bar{u}_{R} \geq 0$ in $T_{R}$.

Proof. We first check that $\partial_{y} \bar{u}_{R} \geq 0$ on $\partial T_{R}$. To see this, simply note that $\bar{u}_{R} \equiv 0$ on the part of the boundary in the Simons cone, $\{t=s<R\}$. Since $\partial_{y}$ is a tangential derivative here, we have $\partial_{y} \bar{u}_{R}=0$ in $\{t=s<R\}$. Take now a point $(s=R, t)$ with $0<t<R$ on the remaining part of the boundary. Recall that $\bar{u}_{R} \leq u_{0}(z)$ in all $T_{R}$. Thus, for all $0<\delta<t$, we have $\bar{u}_{R}(R-\delta, t-\delta) \leq u_{0}((R-\delta-(t-\delta)) / \sqrt{2})=u_{0}((R-t) / \sqrt{2})=\bar{u}_{R}(R, t)$. Differentiating with respect to $\delta$ at $\delta=0$, we deduce $\partial_{y} \bar{u}_{R}(R, t) \geq 0$.

Thus $\partial_{y} \bar{u}_{R} \geq 0$ on $\partial T_{R}$ and hence we can take $\left(\partial_{y} \bar{u}_{R}\right)^{-}$as a test function in $Q_{\bar{u}_{R}}$.

As in (3.11), we now use the analogue equation for $\partial_{s} \bar{u}_{R}$ :

$$
-\Delta \partial_{s} \bar{u}_{R}-f^{\prime}\left(\bar{u}_{R}\right) \partial_{s} \bar{u}_{R}=-\frac{m-1}{s^{2}} \partial_{s} \bar{u}_{R} \quad \text { in } T_{R}
$$

From this and (3.11), since $\partial_{y}=\left(\partial_{s}+\partial_{t}\right) / \sqrt{2}$, we obtain

$$
\begin{array}{r}
-\Delta \partial_{y} \bar{u}_{R}-f^{\prime}\left(\bar{u}_{R}\right) \partial_{y} \bar{u}_{R}=-\frac{m-1}{\sqrt{2}}\left(\frac{\partial_{s} \bar{u}_{R}}{s^{2}}+\frac{\partial_{t} \bar{u}_{R}}{t^{2}}\right) \\
=-\frac{m-1}{s^{2}} \partial_{y} \bar{u}_{R}-\frac{(m-1)\left(s^{2}-t^{2}\right)}{\sqrt{2} s^{2} t^{2}} \partial_{t} \bar{u}_{R}
\end{array}
$$

in $T_{R} \cap\{t>0\}$. Thus,

$$
-\Delta\left(-\partial_{y} \bar{u}_{R}\right)-f^{\prime}\left(\bar{u}_{R}\right)\left(-\partial_{y} \bar{u}_{R}\right)=-\frac{m-1}{s^{2}}\left(-\partial_{y} \bar{u}_{R}\right)+\frac{(m-1)\left(s^{2}-t^{2}\right)}{\sqrt{2} s^{2} t^{2}} \partial_{t} \bar{u}_{R}
$$

in $T_{R} \cap\{t>0\}$.

Multiplying (3.12) by $\left(\partial_{y} \bar{u}_{R}\right)^{-}$and integrating by parts, we have that

$$
\begin{aligned}
0 \leq & Q_{\bar{u}_{R}}\left(\left(\partial_{y} \bar{u}_{R}\right)^{-}\right) \\
= & \int_{T_{R}}\left\{\left|\nabla\left(\partial_{y} \bar{u}_{R}\right)^{-}\right|^{2}-f^{\prime}\left(\bar{u}_{R}\right)\left(\left(\partial_{t} \bar{u}_{R}\right)^{-}\right)^{2}\right\} d x \\
= & -\int_{T_{R}} \frac{m-1}{s^{2}}\left(\left(\partial_{y} \bar{u}_{R}\right)^{-}\right)^{2} d x+ \\
& \quad+\int_{T_{R}} \frac{(m-1)\left(s^{2}-t^{2}\right)}{\sqrt{2} s^{2} t^{2}} \partial_{t} \bar{u}_{R}\left(\partial_{y} \bar{u}_{R}\right)^{-} d x \leq 0,
\end{aligned}
$$

since $\partial_{t} \bar{u}_{R} \leq 0$ in $T_{R}$ by Lemma 3.9 . Therefore $\left(\partial_{y} \bar{u}_{R}\right)^{-} \equiv 0$ in $T_{R}$, which finishes the proof.

Finally we prove Theorem 1.7 
Proof of Theorem 1.7. By Propositions 3.8 and 3.5 we know that there exist a positive minimal solution $\underline{u}$ and a positive maximal solution $\bar{u}$ in $\mathcal{O}$ in the sense stated in the propositions. In addition, they depend only on $s$ and $t$.

Now, since $f$ is odd, by odd reflection with respect to $\mathcal{C}$ we obtain saddle solutions $\underline{u}$ and $\bar{u}$ in $\mathbb{R}^{2 m}$ such that

$$
|\underline{u}| \leq|u| \leq|\bar{u}| \quad \text { in } \mathbb{R}^{2 m},
$$

for every solution $u$ of $-\Delta u=f(u)$ in $\mathbb{R}^{2 m}$ that vanishes on the Simons cone and has the same sign as $s-t$.

It only remains to prove the monotonicity properties stated in the theorem. First, since $\bar{u}$ is the limit of $\bar{u}_{R}$ as $R \rightarrow \infty$, Lemmas 3.9] and 3.10 give that $-\partial_{t} \bar{u} \geq 0$ and $\partial_{y} \bar{u} \geq 0$ in $\{s>t\}$. As a consequence, $\partial_{s} \bar{u} \geq 0$ in $\{s>t\}$.

It follows, since $u(s, t)=-u(t, s)$, that $-\partial_{t} \bar{u} \geq 0$ in all of $\mathbb{R}^{2 m}$. Our equation reads

$$
-\partial_{s s} \bar{u}-\partial_{t t} \bar{u}-(m-1) \frac{\partial_{s} \bar{u}}{s}-(m-1) \frac{\partial_{t} \bar{u}}{t}=f(\bar{u}) \text { in } T_{R} \cap\{t>0\}
$$

and, differentiating it with respect to $t$, we also have

$$
-\Delta \partial_{t} \bar{u}-f^{\prime}(\bar{u}) \partial_{t} \bar{u}=-\frac{m-1}{t^{2}} \partial_{t} \bar{u} \quad \text { in } T_{R} \cap\{t>0\} .
$$

Since $-\partial_{t} \bar{u} \geq 0$ in $\mathbb{R}^{2 m},(\underline{3.14})$ and the strong maximum principle give that $-\partial_{t} \bar{u}>0$ in $\mathbb{R}^{2 m} \backslash\{t=0\}$. On the other hand, multiplying (3.13) by $t$, using that every saddle solution is of class $C^{2}$, and letting $t \rightarrow 0$, we deduce $-\partial_{t} \bar{u}=0$ in $\{t=0\}$. Statement (a) of the theorem is now proved.

Part (b) is proved in the same way - or it is simply the symmetric result to (a). Statement (c) follows directly from (a) and (b).

Finally, we prove (d). Equation (3.12), after letting $R \rightarrow \infty$, gives

$$
\begin{aligned}
-\Delta \partial_{y} \bar{u}-f^{\prime}(\bar{u}) \partial_{y} \bar{u} & =-\frac{m-1}{s^{2}} \partial_{y} \bar{u}-\frac{(m-1)\left(s^{2}-t^{2}\right)}{\sqrt{2} s^{2} t^{2}} \partial_{t} \bar{u} \\
& \geq-\frac{m-1}{s^{2}} \partial_{y} \bar{u}
\end{aligned}
$$

in $\{s>t>0\}$, since $\partial_{t} \bar{u} \leq 0$ in this set. We have already proved (using Lemma 3.10) that $\partial_{y} \bar{u} \geq 0$ in $\{s>t\}$. Hence, the strong maximum principle leads to $\partial_{y} \bar{u}>0$ in $\{s>t\}$, as claimed.

Finally we prove Proposition 1.10, which states that every solution that vanishes on the Simons cone $\mathcal{C}$ and has the same sign as $s-t$ is stable in $\mathcal{O}$.

Proof of Proposition 1.10. Let $u$ be a bounded solution of $-\Delta u=f(u)$ in $\mathbb{R}^{2 m}$ that vanishes on the Simons cone $\mathcal{C}=\{s=t\}$ and has the same sign as $s-t$. Then, by Proposition 2.2 , we have that $0<u<M$ in $\mathcal{O}=\{s>t\}$. Thus, by Remark 3.3 , $Q_{u}(\xi) \geq 0$ for all $\xi \in H^{1}\left(\mathbb{R}^{2 m}\right)$ with compact support and such that $\xi \equiv 0$ in $\mathbb{R}^{2 m} \backslash\{s>t\}$, where $Q_{u}$ is defined in (1.10). 
By the analogue (or symmetric) argument now in $\{s<t\}$ (instead of $\{s>t\}$ ), and since $-M<u<0$ in $\{s<t\}$ by Proposition 2.2 , $Q_{u}(\xi) \geq 0$ for all $\xi \in H^{1}\left(\mathbb{R}^{2 m}\right)$ with compact support and such that $\xi \equiv 0$ in $\mathbb{R}^{2 m} \backslash\{s<t\}$.

Now, given $\xi \in C^{1}\left(\mathbb{R}^{2 m}\right)$ with compact support and with $\xi \equiv 0$ in $\mathcal{C}$, we write $\xi=\chi_{\{s>t\}} \xi+\chi_{\{s<t\}} \xi$, the sum of two $H^{1}$ functions, and we use the previous facts to conclude $Q_{u}(\xi) \geq 0$.

\section{Asymptotic Behavior of SAdDle SOlutions in $\mathbb{R}^{2 m}$}

This section is devoted to study the asymptotic behavior at infinity of saddle-shaped solutions to $-\Delta u=f(u)$ in $\mathbb{R}^{2 m}$, and more generally, of solutions (not necessarily depending only on $s$ and $t$ ) which are odd with respect to the Simons cone $\mathcal{C}$ and positive in $\mathcal{O}=\{s>t\}$.

We will work in the $(y, z)$ system of coordinates. Recall that we defined, in (1.14), $y$ and $z$ by

$$
\left\{\begin{array}{l}
y=(s+t) / \sqrt{2} \\
z=(s-t) / \sqrt{2}
\end{array}\right.
$$

which satisfy $y \geq 0$ and $-y \leq z \leq y$.

We prove Theorem [1.6, which states that any solution $u$ as above tends at infinity to the function

$$
U(x):=u_{0}(z)
$$

uniformly outside of compact sets. Similarly the gradient of $u, \nabla u$, converges to $\nabla U$. This fact will be important in the proof of instability of saddle solutions in dimension 6 .

Our proof of Theorem [1.6, which argues by contradiction, uses a well known compactness argument based on translations of the solution, as well as two crucial classification or Liouville type results for monostable equations. Regarding the nonlinearity, both assume that $g:[0,+\infty) \rightarrow \mathbb{R}$ is a $C^{1}$ function such that

$$
g(0)=g(1)=0, g^{\prime}(0)>0, g>0 \text { in }(0,1), \text { and } g<0 \text { in }(1,+\infty) .
$$

The first result concerns global solutions, that is solutions in all space, and is originally due to Aronson and Weinberger [7]; the statement that we present, a simpler proof, and much more general results are due to Berestycki, Hamel, and Nadirashvili, see Proposition 1.14 of [10] (see also [11] for more general results).

Proposition 4.1. (Aronson-Weinberger [7]; Berestycki-Hamel-Nadirashvili [10]). Let $g$ satisfy (4.1) and let $b \in \mathbb{R}^{n}$ be such that $|b|<2 \sqrt{g^{\prime}(0)}$. Let $u$ be a bounded solution of

$$
\left\{\begin{aligned}
\Delta u+b \cdot \nabla u+g(u) & =0 & & \text { in } \mathbb{R}^{n} \\
u & \geq 0 & & \text { in } \mathbb{R}^{n} .
\end{aligned}\right.
$$

Then, $u \equiv 0$ or $u \equiv 1$. 
The second result concerns the same equation but in a half-space. It states the 1D symmetry of its solutions and is due to Angenent [6]. For Lipschitz nonlinearities and slightly weaker assumptions on them, see BerestyckiCaffarelli-Nirenberg [8].

Proposition 4.2 (Angenent [6]). Let $g$ satisfy (4.1) and also $g^{\prime}(1)<0$. Let $u$ be a bounded solution of

$$
\left\{\begin{aligned}
\Delta u+g(u)=0 & \text { in } \mathbb{R}_{+}^{n}=\left\{x_{n}>0\right\} \\
u=0 & \text { on } \partial \mathbb{R}_{+}^{n} \\
u>0 & \text { in } \mathbb{R}_{+}^{n} .
\end{aligned}\right.
$$

Then, $u$ depends only on the $x_{n}$ variable. In addition, such solution depending only on $x_{n}$ exists and is unique.

The two previous propositions use Serrin's sweeping method as important tool in their proofs (see [6] and Remark 4.3 of [10]).

A very simple lemma that we will use in the proof of Theorem 1.6 is the following (see Lemma 4.2 of [14] and its proof there).

Lemma 4.3 ([14]). For every $x=\left(x^{1}, x^{2}\right) \in \mathbb{R}^{2 m}$, the distance from $x$ to the Simons cone $\mathcal{C}$ is given by $\operatorname{dist}(x, \mathcal{C})=|s-t| / \sqrt{2}$, where $s=\left|x^{1}\right|$ and $t=\left|x^{2}\right|$. In addition, if $s=\left|x^{1}\right| \neq 0$ and $t=\left|x^{2}\right| \neq 0$, then $\operatorname{dist}(x, \mathcal{C})=\left|x-x_{0}\right|$ where $x_{0} \in \mathcal{C}$ has coordinates

$$
x_{0}=\left(\alpha x^{1}, \beta x^{2}\right)
$$

and $\alpha$ and $\beta$ are defined by $\alpha s=\beta t=(s+t) / 2$.

Proof of Theorem 1.6. Suppose that the theorem does not hold. Thus, let $u$ be a solution of $-\Delta u=f(u)$ in $\mathbb{R}^{2 m}$ that vanishes on the cone $\mathcal{C}$, is positive in the region $\mathcal{O}=\{s>t\}$ and odd with respect to $\mathcal{C}$, and for which there exists $\varepsilon>0$ and a sequence $\left\{x_{k}\right\}$ with

$$
\left|x_{k}\right| \rightarrow \infty \quad \text { and } \quad\left|u\left(x_{k}\right)-U\left(x_{k}\right)\right|+\left|\nabla u\left(x_{k}\right)-\nabla U\left(x_{k}\right)\right|>\varepsilon .
$$

By continuity we may move slightly $x_{k}$ and assume $x_{k} \notin \mathcal{C}$ for all $k$. Moreover, up to a subsequence (which we still denote by $\left\{x_{k}\right\}$ ), either $\left\{x_{k}\right\} \subset\{s>t\}$ or $\left\{x_{k}\right\} \subset\{s<t\}$. By the symmetries of the problem we may assume $\left\{x_{k}\right\} \subset\{s>t\}=\mathcal{O}$.

Recall that, by Proposition 2.2, we know that $|u(x)| \leq\left|u_{0}(z)\right|<M$ in $\mathbb{R}^{2 m}$. We distinguish two cases:

CASE 1. $\left\{\operatorname{dist}\left(x_{k}, \mathcal{C}\right)=: d_{k}\right\}$ is an unbounded sequence.

In this case, since $0<z_{k}=\operatorname{dist}\left(x_{k}, \mathcal{C}\right)=d_{k} \rightarrow+\infty$ (for a subsequence), we have that $U\left(x_{k}\right)=u_{0}\left(z_{k}\right)=u_{0}\left(d_{k}\right)$ tends to $M$ and $\left|\nabla U\left(x_{k}\right)\right|$ tends to 0 , that is,

$$
U\left(x_{k}\right) \rightarrow M \quad \text { and } \quad\left|\nabla U\left(x_{k}\right)\right| \rightarrow 0 .
$$

From this and (4.2) we have

$$
\left|u\left(x_{k}\right)-M\right|+\left|\nabla u\left(x_{k}\right)\right| \geq \frac{\varepsilon}{2},
$$


for $k$ large enough.

Consider the ball $B_{d_{k}}(0)$ of radius $d_{k}$ centered at 0 , and define

$$
v_{k}(\tilde{x})=u\left(\tilde{x}+x_{k}\right), \quad \text { for } \tilde{x} \in B_{d_{k}}(0) .
$$

Since $B_{d_{k}}(0)+x_{k} \subset\{s>t\}$ we have that $0<v_{k}<M$ in $B_{d_{k}}(0)$ and $-\Delta v_{k}=f\left(v_{k}\right)$ in $B_{d_{k}}(0)$. Letting $k$ tend to infinity we obtain, through a subsequence, a solution $v$ to the problem in all of $\mathbb{R}^{2 m}$. That is, $v$ satisfies

$$
\left\{\begin{aligned}
-\Delta v=f(v) \quad \text { in } \mathbb{R}^{2 m} \\
v \geq 0 \quad \text { in } \mathbb{R}^{2 m} .
\end{aligned}\right.
$$

Define $w=v / M$ where $M$ is given by the assumptions on $f$. Then $w$ is a nonnegative solution of $-\Delta w=g(w)$ in $\mathbb{R}^{2 m}$, where $g(w)=\frac{1}{M} f(M w)$. Since $w \leq 1$ in $\mathbb{R}^{2 m}$, we may change $g$ in $(1,+\infty)$ to be negative here (for instance, taking $g$ linear in $(1,+\infty)$ with slope $g^{\prime}(1)<0$ and $\left.g(1)=0\right)$, and $w$ is still a solution of the same equation. Since $f$ satisfies (1.9), the function $g$ (changed as above in $(1,+\infty))$ satisfies the conditions of Proposition 4.1 with $b \equiv 0$. Hence we have that $w \equiv 0$ or $w \equiv 1$, that is, $v \equiv 0$ or $v \equiv M$. In either case, $\nabla v(0)=0$, that is, $\left|\nabla u\left(x_{k}\right)\right|$ tends to 0 .

Next we show that $v \not \equiv 0$. By Proposition 1.10 (established in the previous section), we have that $u$ is stable in $\mathcal{O}$. Hence, $v_{k}$ is semi-stable in $B_{d_{k}}(0)$ (since $\left.B_{d_{k}}(0)+x_{k} \subset \mathcal{O}\right)$ in the sense of Definition 3.2. This implies that $v$ is stable in all of $\mathbb{R}^{2 m}$ and therefore $v \not \equiv 0$ (otherwise, since $f^{\prime}(0)>0$ we could construct a test function $\xi$ such that $Q_{v}(\xi)<0$ which would be a contradiction with the fact that $v$ is stable).

Hence, it must be $v \equiv M$. But this implies that $v(0)=M$ and so $u\left(x_{k}\right)$ tends to $M$. Hence, we have that $u\left(x_{k}\right)$ tends to $M$ and $\left|\nabla u\left(x_{k}\right)\right|$ tends to 0 , which is a contradiction with (4.3). Therefore, we have proved the theorem in this case 1.

CASE 2. $\left\{\operatorname{dist}\left(x_{k}, \mathcal{C}\right)=: d_{k}\right\}$ is a bounded sequence.

The points $x_{k}$ remain at a finite distance to the cone. Then, at least for a subsequence, $d_{k} \rightarrow d \geq 0$ as $k \rightarrow \infty$. Let $x_{k}^{0} \in \mathcal{C}$ be a point that realizes the distance to the cone, that is,

$$
\operatorname{dist}\left(x_{k}, \mathcal{C}\right)=\left|x_{k}-x_{k}^{0}\right|=d_{k},
$$

and let $\nu_{k}^{0}$ be the inner unit normal to $\mathcal{C}=\partial \mathcal{O}$ at $x_{k}^{0}$. Note that $B_{d_{k}}\left(x_{k}\right) \subset$ $\mathcal{O} \subset \mathbb{R}^{2 m} \backslash \mathcal{C}$ and $x_{k}^{0} \in \partial B_{d_{k}}\left(x_{k}\right) \cap \mathcal{C}$, i.e., $x_{k}^{0}$ is the point where the sphere $\partial B_{d_{k}}\left(x_{k}\right)$ is tangent to the cone $\mathcal{C}$. It follows that $x_{k}^{0} \neq 0$ and also that $\left(x_{k}-x_{k}^{0}\right) / d_{k}$ is the unit normal $\nu_{k}^{0}$ to $\mathcal{C}$ at $x_{k}^{0}$. That is, $x_{k}=x_{k}^{0}+d_{k} \nu_{k}^{0}$. This will be checked below, in an alternative way, with explicit formulae. Now, since the sequence $\left\{\nu_{k}^{0}\right\}$ is bounded, there exists a subsequence such that

$$
\nu_{k}^{0} \rightarrow \nu \in \mathbb{R}^{2 m}, \quad|\nu|=1
$$

We define

$$
w_{k}(\tilde{x})=u\left(\tilde{x}+x_{k}^{0}\right) \quad \text { for } \tilde{x} \in \mathbb{R}^{2 m}
$$


The functions $w_{k}$ are all solutions of $-\Delta w=f(w)$ in $\mathbb{R}^{2 m}$ and are uniformly bounded. Hence, by interior elliptic estimates the sequence $\left\{w_{k}\right\}$ converges locally in $C^{2}$, up to a subsequence, to a solution in $\mathbb{R}^{2 m}$. Therefore we have that, as $k$ tends to infinity and up to a subsequence,

$$
w_{k} \rightarrow w \text { and } \nabla w_{k} \rightarrow \nabla w \text { uniformly on compact sets of } \mathbb{R}^{2 m},
$$

where $w$ is a solution of $-\Delta w=f(w)$ in $\mathbb{R}^{2 m}$. Note that the curvature of $\mathcal{C}$ at $x_{k}^{0}$ goes to zero as $k$ tends to infinity, since $\mathcal{C}$ is a cone and $\left|x_{k}^{0}\right| \rightarrow \infty$ (note that $\left|x_{k}^{0}\right| \rightarrow \infty$ due to $\left|x_{k}\right| \rightarrow \infty$ and $\left|x_{k}-x_{k}^{0}\right|=d_{k} \rightarrow d<\infty$ ). Thus, $\mathcal{C}$ at $x_{k}^{0}$ is flatter and flatter as $k \rightarrow \infty$ and since we translate $x_{k}^{0}$ to 0 , the limiting function $w$ satisfies (all this we will prove below in detail)

$$
\left\{\begin{aligned}
\Delta w+f(w)=0 & \text { in } H:=\{\tilde{x} \cdot \nu>0\} \\
w=0 & \text { on } \partial H \\
w>0 & \text { in } H
\end{aligned}\right.
$$

a problem in a half-space.

Now, by Proposition 1.10, $u$ is stable for perturbations with compact support in $\mathcal{O}$, and thus $w_{k}$ (for $k$ large) and $w$ are stable for perturbations with compact support in $H$-see the computation in (4.9) below for details. Therefore $w$ can not be identically zero. By assumption (1.9) on $f$, we can apply Proposition 4.2 above (applied to $w / M$ to have $M=1$ ), and deduce that $w$ is the unique $1 \mathrm{D}$ solution, that is, the solution depending only on one variable (the orthogonal direction to $\partial H$ ). Hence,

$$
w(\tilde{x})=u_{0}(\tilde{x} \cdot \nu) \quad \text { for all } \tilde{x} \in H .
$$

From the definition of $w_{k}$, and using that $z_{k}=d_{k}=\left|x_{k}-x_{k}^{0}\right|$ is a bounded sequence and that $x_{k}-x_{k}^{0}=d_{k} \nu_{k}^{0}$, we have that

$$
\begin{aligned}
u\left(x_{k}\right) & =w_{k}\left(x_{k}-x_{k}^{0}\right)=w\left(x_{k}-x_{k}^{0}\right)+\mathrm{o}(1)=u_{0}\left(\left(x_{k}-x_{k}^{0}\right) \cdot \nu\right)+\mathrm{o}(1) \\
& =u_{0}\left(\left(x_{k}-x_{k}^{0}\right) \cdot \nu_{k}^{0}\right)+\mathrm{o}(1)=u_{0}\left(d_{k}\left|\nu_{k}^{0}\right|^{2}\right)+\mathrm{o}(1) \\
& =u_{0}\left(z_{k}\right)+\mathrm{o}(1)=U\left(x_{k}\right)+\mathrm{o}(1) .
\end{aligned}
$$

The same argument can be done for $\nabla u\left(x_{k}\right)$ and $\nabla U\left(x_{k}\right)$. We arrive to a contradiction with (4.2).

Finally, we prove in detail the statements in (4.6): $w>0$ in $H$ and $w=0$ on $\partial H$, as well as the identity $x_{k}-x_{k}^{0}=d_{k} \nu_{k}^{0}$ used before.

Let $x_{k} \in \mathcal{O}$ and $x_{k}^{0} \in \mathcal{C}$ as in (4.5). Since $\left|x_{k}\right| \rightarrow \infty$ and $\operatorname{dist}\left(x_{k}, \mathcal{C}\right)=$ $\left|s_{k}-t_{k}\right| / \sqrt{2}$ remains bounded, we have $s_{k} \neq 0$ and $t_{k} \neq 0$ for $k$ large enough. Thus, by Lemma 4.3 we may write the coordinates of $x_{k}^{0}$ as

$$
x_{k}^{0}=\left(\alpha x_{k}^{1}, \beta x_{k}^{2}\right), \quad \text { for } \quad 2 \alpha s_{k}=2 \beta t_{k}=s_{k}+t_{k} .
$$

In particular, $\left|x_{k}^{0}\right| \neq 0$. Then, since we are assuming, without loss of generality, that $x_{k} \in \mathcal{O}=\{s>t\}$ we have that

$$
d_{k}=\left|x_{k}-x_{k}^{0}\right|=\frac{s_{k}-t_{k}}{\sqrt{2}} .
$$


Since $\mathcal{C}=\left\{s^{2}-t^{2}=0\right\}$ we can write the coordinates of $\nu_{k}^{0}$ as

$$
\nu_{k}^{0}=\left(\frac{x_{k}^{0,1}}{\left|x_{k}^{0}\right|}, \ldots, \frac{x_{k}^{0, m}}{\left|x_{k}^{0}\right|},-\frac{x_{k}^{0, m+1}}{\left|x_{k}^{0}\right|}, \ldots,-\frac{x_{k}^{0,2 m}}{\left|x_{k}^{0}\right|}\right) .
$$

Using the previous identities, we conclude that

$$
x_{k}-x_{k}^{0}=d_{k} \nu_{k}^{0} \quad \text { with } \quad \nu_{k}^{0}=\left(\frac{\alpha x_{k}^{1}}{\left|x_{k}^{0}\right|},-\frac{\beta x_{k}^{2}}{\left|x_{k}^{0}\right|}\right) .
$$

Next, for all $\tilde{x} \in \mathbb{R}^{2 m}$,

$$
\tilde{x} \cdot \nu_{k}^{0}=\sum_{i=1}^{m} \frac{x_{k}^{0, i} \tilde{x}_{i}}{\left|x_{k}^{0}\right|}-\sum_{j=m+1}^{2 m} \frac{x_{k}^{0, j} \tilde{x}_{j}}{\left|x_{k}^{0}\right|}=\frac{x_{k}^{0, i} \tilde{x}_{i}-x_{k}^{0, j} \tilde{x}_{j}}{\left|x_{k}^{0}\right|} .
$$

Since $|\nabla u| \in L^{\infty}\left(\mathbb{R}^{2 m}\right)$ and $u\left(x_{k}^{0}\right)=0$,

$$
\left|w_{k}(\tilde{x})\right|=\left|u\left(x_{k}^{0}+\tilde{x}\right)\right| \leq C \operatorname{dist}\left(x_{k}^{0}+\tilde{x}, \mathcal{C}\right) .
$$

From Lemma 4.3 we have that $\operatorname{dist}(x, \mathcal{C})=\left|s_{x}-t_{x}\right| / \sqrt{2}$. Recall also that $t_{x_{k}^{0}}=s_{x_{k}^{0}}$. Now, using (4.7) and sums over repetead indices $i=1, \ldots, m$ and $j=m+1, \ldots, 2 m$ as before, we have

$$
\begin{aligned}
& \operatorname{dist}\left(x_{k}^{0}+\tilde{x}, \mathcal{C}\right)=\frac{1}{\sqrt{2}}\left|\sqrt{s_{x_{k}^{0}}^{2}+s_{\tilde{x}}^{2}+2 x_{k}^{0, i} \tilde{x}_{i}}-\sqrt{s_{x_{k}^{0}}^{2}+t_{\tilde{x}}^{2}+2 x_{k}^{0, j} \tilde{x}_{j}}\right| \\
& =\frac{1}{\sqrt{2}}\left|\frac{s_{\tilde{x}}^{2}-t_{\tilde{x}}^{2}+2\left|x_{k}^{0}\right| \tilde{x} \cdot \nu_{k}^{0}}{\sqrt{s_{x_{k}^{0}}^{2}+s_{\tilde{x}}^{2}+2 x_{k}^{0, i} \tilde{x}_{i}}+\sqrt{s_{x_{k}^{0}}^{2}+t_{\tilde{x}}^{2}+2 x_{k}^{0, j} \tilde{x}_{j}}}\right| \\
& =\mid \frac{1}{\sqrt{2}} \frac{s_{\tilde{x}}^{2}-t_{\tilde{x}}^{2}}{\left|x_{k}^{0}\right|\left(\sqrt{\frac{1}{2}+\frac{s_{\tilde{x}}^{2}}{\left|x_{k}^{0}\right|^{2}}+\frac{2 x_{k}^{0, i} \tilde{x}_{i}}{\left|x_{k}^{0}\right|^{2}}}+\sqrt{\frac{1}{2}+\frac{t_{\tilde{\tilde{x}}}^{2}}{\left|x_{k}^{0}\right|^{2}}+\frac{2 x_{k}^{0, j} \tilde{x}_{j}}{\left|x_{k}^{0}\right|^{2}}}\right)} \\
& +\frac{2}{\sqrt{2}} \frac{\tilde{x} \cdot \nu_{k}^{0}}{\sqrt{\frac{1}{2}+\frac{s_{\tilde{x}}^{2}}{\left|x_{k}^{0}\right|^{2}}+\frac{2 x_{k}^{0, i} \tilde{x}_{i}}{\left|x_{k}^{0}\right|^{2}}}+\sqrt{\frac{1}{2}+\frac{t_{\tilde{x}}^{2}}{\left|x_{k}^{0}\right|^{2}}+\frac{2 x_{k}^{0, j} \tilde{x}_{j}}{\left|x_{k}^{0}\right|^{2}}}} \text {. }
\end{aligned}
$$

Now we let $k$ tend to infinity. Since $\tilde{x}$ is fixed, $\left|x_{k}^{0}\right| \rightarrow \infty$ and $\nu_{k}^{0} \rightarrow \nu$, we obtain

$$
\operatorname{dist}\left(x_{k}^{0}+\tilde{x}, \mathcal{C}\right) \rightarrow|\tilde{x} \cdot \nu| \quad \text { as } k \rightarrow \infty .
$$

Thus, since $w_{k}(\tilde{x}) \rightarrow w(\tilde{x})$, from (4.8) we have that $w(\tilde{x})=0$ for every $\tilde{x} \in \partial H=\{\tilde{x} \cdot \nu=0\}$.

Let now $\tilde{x} \in H$, i.e, $\tilde{x} \cdot \nu>0$. We will prove that $w(\tilde{x}) \geq 0$. We show that for $k$ large, $w_{k}(\tilde{x})=u\left(x_{k}^{0}+\tilde{x}\right) \geq 0$. Since $u$ is positive in $\mathcal{O}=\{s>t\}$, 
we need to establish that $s_{x_{k}^{0}+\tilde{x}} \geq t_{x_{k}^{0}+\tilde{x}}$ for $k$ large. As above, we have that

$$
\begin{aligned}
s_{x_{k}^{0}+\tilde{x}}-t_{x_{k}^{0}+\tilde{x}} & =\sqrt{s_{x_{k}^{0}}^{2}+s_{\tilde{x}}^{2}+2 x_{k}^{0, i} \tilde{x}_{i}}-\sqrt{s_{x_{k}^{0}}^{2}+t_{\tilde{x}}^{2}+2 x_{k}^{0, j} \tilde{x}_{j}} \\
& =\frac{s_{\tilde{x}}^{2}-t_{\tilde{x}}^{2}+2\left|x_{k}^{0}\right| \tilde{x} \cdot \nu_{k}^{0}}{\sqrt{s_{x_{k}^{0}}^{2}+s_{\tilde{x}}^{2}+2 x_{k}^{0, i} \tilde{x}_{i}}+\sqrt{s_{x_{k}^{0}}^{2}+t_{\tilde{x}}^{2}+2 x_{k}^{0, j} \tilde{x}_{j}}},
\end{aligned}
$$

and, letting $k$ tend to infinity, since $\tilde{x}$ is fixed and $\left|x_{k}^{0}\right| \rightarrow \infty$, we obtain

$$
s_{x_{k}^{0}+\tilde{x}}-t_{x_{k}^{0}+\tilde{x}} \rightarrow \sqrt{2} \tilde{x} \cdot \nu>0 \quad \text { as } k \rightarrow \infty .
$$

Therefore $w(\tilde{x}) \geq 0$.

\section{InstabiLity IN DIMENSION 6}

In this section we prove Theorem 1.9, establishing that saddle solutions in dimension 6 are unstable outside of every compact set. The asymptotic analysis done for $n=4$ in the proof of the instability theorem of [14] does not lead to the instability of saddle solutions in dimensions $n \geq 6$. Indeed, the supersolution $u_{0}(z)=u_{0}((s-t) / \sqrt{2})$ is, in every dimension $n=2 m \geq 6$, asymptotically stable at infinity in some weak sense and with respect to perturbations $\xi(y, z)$ with separate variables.

Hence, the proof of instability in dimension 6 requires a more precise argument. We use the equation satisfied by $\bar{u}_{z}$, where $\bar{u}$ is the maximal saddle solution, as well as the monotonicity and asymptotic properties of $\bar{u}$. We prove that $\bar{u}$ is unstable outside of every compact set by constructing test functions $\xi(y, z)=\eta(y) \bar{u}_{z}(y, z)$ such that $Q_{\bar{u}}(\xi)<0$. Since $\bar{u}$ is maximal, its instability outside of compact sets implies that the same instability property holds for all bounded solutions $u$ vanishing on the Simons cone $\mathcal{C}$ and positive in $\mathcal{O}$.

Recall that a bounded solution $u$ of $-\Delta u=f(u)$ in $\mathbb{R}^{2 m}$ is stable provided

$$
Q_{u}(\xi)=\int_{\mathbb{R}^{2 m}}\left\{|\nabla \xi|^{2}-f^{\prime}(u) \xi^{2}\right\} d x \geq 0 \quad \text { for all } \xi \in C_{c}^{\infty}\left(\mathbb{R}^{2 m}\right) .
$$

If $v$ is a function depending only on $s$ and $t$, the quadratic form $Q_{v}(\xi)$ acting on perturbations of the form $\xi=\xi(s, t)$ becomes

$$
c_{m} Q_{v}(\xi)=\int_{\{s>0, t>0\}} s^{m-1} t^{m-1}\left\{\xi_{s}^{2}+\xi_{t}^{2}-f^{\prime}(v) \xi^{2}\right\} d s d t,
$$

where $c_{m}>0$ is a constant depending only on $m$. We can further change to variables $(y, z)$ and obtain, for a different constant $c_{m}>0$,

$$
c_{m} Q_{v}(\xi)=\int_{\{-y<z<y\}}\left(y^{2}-z^{2}\right)^{m-1}\left\{\xi_{y}^{2}+\xi_{z}^{2}-f^{\prime}(v) \xi^{2}\right\} d y d z .
$$

Given the definition of the variables $y$ and $z$, a function $\xi=\xi(y, z)$ has compact support in $\mathbb{R}^{2 m}$ if and only if $\xi(y, z)$ vanishes for $y$ large enough. 
Now let $u$ be a bounded solution of $-\Delta u=f(u)$ in $\mathbb{R}^{2 m}$ vanishing on the Simons cone $\mathcal{C}=\{s=t\}$ and positive in $\mathcal{O}=\{s>t\}$. By Theorem 1.7, we know that

$$
|u(x)| \leq|\bar{u}(x)| \quad \text { in all of } \mathbb{R}^{2 m} .
$$

This leads to $f^{\prime}(|u(x)|) \geq f^{\prime}(|\bar{u}(x)|)$ for all $x \in \mathbb{R}^{2 m}$, since we assume $f$ to be concave in $(0, M)$. Now, since $f^{\prime}$ is even, we deduce that

$$
f^{\prime}(u(x)) \geq f^{\prime}(\bar{u}(x)) \quad \text { for all } x \in \mathbb{R}^{2 m} .
$$

Therefore, we conclude

$$
Q_{u}(\xi) \leq Q_{\bar{u}}(\xi) \quad \text { for all } \xi \in C_{c}^{\infty}\left(\mathbb{R}^{2 m}\right) .
$$

It follows that, in order to prove that $u$ is unstable, it suffices to find a smooth function $\xi$ with compact support in $\mathbb{R}^{2 m}$ for which $Q \bar{u}(\xi)<0$. Note also that, by an approximation argument, it suffices to find a Lipschitz function $\xi=\xi(y, z)$, not necessarily smooth, vanishing for all $y \in(0,+\infty) \backslash I$, where $I$ is a compact interval in $(0,+\infty)$, and for which $Q_{\bar{u}}(\xi)<0$.

Proof of Theorem 1.9. By the previous arguments, it suffices to establish that the maximal solution $\bar{u}$, whose existence is given by Theorem 1.7, is unstable outside of every compact set.

We have, for every test function $\xi$,

$$
Q_{\bar{u}}(\xi)=\int_{\mathbb{R}^{2 m}}\left(|\nabla \xi|^{2}-f^{\prime}(\bar{u}) \xi^{2}\right) d x .
$$

Suppose now that $\xi=\xi(y, z)=\eta(y, z) \psi(y, z)$, where $\eta$ and $\psi$ are Lipschitz functions, and $\eta(y, z)=0$ whenever $y \in(0,+\infty) \backslash I$, with $I$ a compact interval in $(0,+\infty)$. The expression for $Q_{\bar{u}}$ becomes, since $|\nabla \xi|^{2}=\xi_{y}^{2}+\xi_{z}^{2}$,

$$
Q \bar{u}(\xi)=\int_{\mathbb{R}^{2 m}}\left(|\nabla \eta|^{2} \psi^{2}+\eta^{2}|\nabla \psi|^{2}+2 \eta \psi \nabla \eta \cdot \nabla \psi-f^{\prime}(\bar{u}) \eta^{2} \psi^{2}\right) d x .
$$

Integrating by parts and using that $2 \eta \psi \nabla \eta \cdot \nabla \psi=\psi \nabla\left(\eta^{2}\right) \cdot \nabla \psi$, we have

$$
Q_{\bar{u}}(\xi)=\int_{\mathbb{R}^{2 m}}\left(|\nabla \eta|^{2} \psi^{2}-\eta^{2} \psi \Delta \psi-f^{\prime}(\bar{u}) \eta^{2} \psi^{2}\right) d x
$$

that is,

$$
Q \bar{u}(\xi)=\int_{\mathbb{R}^{2 m}}\left(|\nabla \eta|^{2} \psi^{2}-\eta^{2} \psi\left(\Delta \psi+f^{\prime}(\bar{u}) \psi\right)\right) d x .
$$

Choose now $\psi(y, z)=\bar{u}_{z}(y, z)$. Since $\bar{u}$ is a saddle solution of $-\Delta \bar{u}=f(\bar{u})$ we may differentiate this equation written in $(y, z)$ variables - see (1.15) with respect to $z$ and find

$$
0=\Delta \bar{u}_{z}+f^{\prime}(\bar{u}) \bar{u}_{z}-\frac{2(m-1)}{y^{2}-z^{2}} \bar{u}_{z}+\frac{4(m-1) z}{\left(y^{2}-z^{2}\right)^{2}}\left(y \bar{u}_{y}-z \bar{u}_{z}\right) .
$$

Replacing in the expression for $Q_{\bar{u}}$ we obtain,

$$
Q_{\bar{u}}(\xi)=\int_{\mathbb{R}^{2 m}}\left(|\nabla \eta|^{2} \bar{u}_{z}^{2-}\right.
$$




$$
\left.-\eta^{2}\left\{\frac{2(m-1)\left(y^{2}+z^{2}\right)}{\left(y^{2}-z^{2}\right)^{2}} \bar{u}_{z}^{2}-\frac{4(m-1) z y}{\left(y^{2}-z^{2}\right)^{2}} \bar{u}_{y} \bar{u}_{z}\right\}\right) d x .
$$

Next we change coordinates to $(y, z)$ and as in (5.1) we have, for some positive constant $c_{m}$,

$$
\begin{aligned}
c_{m} Q_{\bar{u}}(\xi)=\int_{\{-y<z<y\}}\left(y^{2}-z^{2}\right)^{m-1}\left(|\nabla \eta|^{2} \bar{u}_{z}^{2}-\right. & \\
& \left.\quad-\eta^{2}\left\{\frac{2(m-1)\left(y^{2}+z^{2}\right)}{\left(y^{2}-z^{2}\right)^{2}} \bar{u}_{z}^{2}-\frac{4(m-1) z y}{\left(y^{2}-z^{2}\right)^{2}} \bar{u}_{y} \bar{u}_{z}\right\}\right) d y d z .
\end{aligned}
$$

For $a>1$, a constant that we will make tend to infinity, let $\eta=\eta(\rho)$ be a Lipschitz function of $\rho:=y / a$ with compact support $\left[\rho_{1}, \rho_{2}\right] \subset(0,+\infty)$. Let us denote by

$$
\eta_{a}(y)=\eta(y / a) \quad \text { and } \quad \xi_{a}(y, z)=\eta_{a}(y) \bar{u}_{z}(y, z)=\eta(y / a) \bar{u}_{z}(y, z),
$$

the functions named $\eta$ and $\xi$ above. The change $y=a \rho, d y=a d \rho$ yields

$$
\begin{aligned}
c_{m} Q_{\bar{u}}\left(\xi_{a}\right)= & a^{2 m-3} \int_{\{-a \rho<z<a \rho\}} \rho^{2(m-1)}\left(1-\frac{z^{2}}{a^{2} \rho^{2}}\right)^{m-1}\left(\eta_{\rho}^{2} \bar{u}_{z}^{2}-\right. \\
& \left.-\eta^{2}\left\{\frac{2(m-1)\left(1+\frac{z^{2}}{a^{2} \rho^{2}}\right)}{\rho^{2}\left(1-\frac{z^{2}}{a^{2} \rho^{2}}\right)^{2}} \bar{u}_{z}^{2}-\frac{4(m-1) z}{a \rho^{3}\left(1-\frac{z^{2}}{a^{2} \rho^{2}}\right)^{2}} \bar{u}_{y} \bar{u}_{z}\right\}\right) d \rho d z .
\end{aligned}
$$

Replacing $m=3$ we get

$$
\begin{aligned}
& \frac{c_{3} Q_{\bar{u}}\left(\xi_{a}\right)}{a^{3}}=\int_{\{-a \rho<z<a \rho\}} \rho^{4}\left(1-\frac{z^{2}}{a^{2} \rho^{2}}\right)^{2}\left(\eta_{\rho}^{2} \bar{u}_{z}^{2}-\right. \\
& \left.-\eta^{2}\left\{\frac{4\left(1+\frac{z^{2}}{a^{2} \rho^{2}}\right)}{\rho^{2}\left(1-\frac{z^{2}}{a^{2} \rho^{2}}\right)^{2}} \bar{u}_{z}^{2}-\frac{8 z}{a \rho^{3}\left(1-\frac{z^{2}}{a^{2} \rho^{2}}\right)^{2}} \bar{u}_{y} \bar{u}_{z}\right\}\right) d \rho d z .
\end{aligned}
$$

We obtain, by using $\left(1-\frac{z^{2}}{a^{2} \rho^{2}}\right)^{2} \leq 1$ and $1+\frac{z^{2}}{a^{2} \rho^{2}} \geq 1$,

$$
\begin{gathered}
\frac{c_{3} Q_{\bar{u}}\left(\xi_{a}\right)}{a^{3}} \leq \int_{\{-a \rho<z<a \rho\}} \rho^{4} \bar{u}_{z}^{2}(a \rho, z)\left(\eta_{\rho}^{2}-\frac{4}{\rho^{2}} \eta^{2}\right) d \rho d z+ \\
\quad+\int_{\{-a \rho<z<a \rho\}} \frac{8 z \rho \eta^{2}(\rho)}{a} \bar{u}_{y}(a \rho, z) \bar{u}_{z}(a \rho, z) d \rho d z .
\end{gathered}
$$

We study these two integrals separately. From Theorem 1.6 we have that $\bar{u}_{y}(a \rho, z) \rightarrow 0$ uniformly, for all $\rho \in\left[\rho_{1}, \rho_{2}\right]=\operatorname{supp} \eta$, as $a$ tends to infinity. Hence, given $\varepsilon>0$, for $a$ sufficiently large, $\left|\bar{u}_{y}(a \rho, z)\right| \leq \varepsilon$. Moreover, by Theorem 1.7 we have that $\bar{u}_{z} \geq 0$ in $\mathbb{R}^{6}$. Hence, since $\eta$ is bounded, for $a$ 
large we have

$$
\begin{aligned}
\left|\int \frac{8 z \rho \eta^{2}(\rho)}{a} \bar{u}_{y} \bar{u}_{z} d \rho d z\right| & \leq \int\left|\frac{8 z \rho \eta^{2}(\rho)}{a}\right|\left|\bar{u}_{y}\right| \bar{u}_{z} d \rho d z \\
& \leq \int 8 \rho^{2} \eta^{2}(\rho)\left|\bar{u}_{y}\right| \bar{u}_{z} d \rho d z \\
& \leq C \varepsilon \int_{\rho_{1}}^{\rho_{2}} \rho^{2} d \rho \int_{-a \rho}^{a \rho} \bar{u}_{z} d z \\
& =C \varepsilon \int_{\rho_{1}}^{\rho_{2}}(\bar{u}(a \rho, a \rho)-\bar{u}(a \rho,-a \rho)) d \rho \\
& \leq C \varepsilon,
\end{aligned}
$$

where $C$ are different constants depending on $\rho_{1}$ and $\rho_{2}$. Hence, as $a$ tends to infinity, this integral converges to zero.

Now, for the other integral we have that again by Theorem 1.6, $\bar{u}_{z}(a \rho, z)$ converges to $\dot{u}_{0}(z)$. We write

$$
\begin{gathered}
\int_{\{-a \rho<z<a \rho\}} \rho^{4} \bar{u}_{z}^{2}(a \rho, z)\left(\eta_{\rho}^{2}-\frac{4}{\rho^{2}} \eta^{2}\right) d \rho d z= \\
=\int_{\{-a \rho<z<a \rho\}} \dot{u}_{0}^{2}(z) \rho^{4}\left(\eta_{\rho}^{2}-\frac{4}{\rho^{2}} \eta^{2}\right) d \rho d z+ \\
+\int_{\{-a \rho<z<a \rho\}} \rho^{4}\left(\bar{u}_{z}(a \rho, z)-\dot{u}_{0}(z)\right)\left(\bar{u}_{z}(a \rho, z)+\dot{u}_{0}(z)\right)\left(\eta_{\rho}^{2}-\frac{4}{\rho^{2}} \eta^{2}\right) d \rho d z .
\end{gathered}
$$

For $a$ large, $\left|\bar{u}_{z}(a \rho, z)-\dot{u}_{0}(z)\right| \leq \varepsilon$ for all $\rho \in\left[\rho_{1}, \rho_{2}\right]$. In addition, $\bar{u}_{z}(a \rho, z)+$ $\dot{u}_{0}(z)$ is positive and is a derivative with respect to $z$ of a bounded function, thus it is integrable in $z$ (by the fundamental theorem of calculus). Hence, since $\eta=\eta(\rho)$ is smooth with compact support, the second integral converges to zero as $a$ tends to infinity. Therefore, letting $a$ tend to infinity, we obtain

$$
\limsup _{a \rightarrow \infty} \frac{c_{3} Q_{\bar{u}}\left(\xi_{a}\right)}{a^{3}} \leq\left(\int_{-\infty}^{+\infty} \dot{u}_{0}^{2}(z) d z\right) \int \rho^{4}\left(\eta_{\rho}^{2}-\frac{4}{\rho^{2}} \eta^{2}\right) d \rho .
$$

Recall that the integral in $d z$ is finite (since $\dot{u}_{0}^{2} \leq C \dot{u}_{0}$ and $\dot{u}_{0}$ is positive and is a derivative of a bounded function).

The integral in $d \rho$ can be seen as an integral in $\mathbb{R}^{5}$ of radial functions $\eta=\eta(\rho)$. Hardy's inequality in $\mathbb{R}^{5}$ states (see e.g. [21]) that

$$
\frac{(5-2)^{2}}{4} \int_{\mathbb{R}^{5}} \frac{\eta^{2}}{|x|^{2}} d x \leq \int_{\mathbb{R}^{5}}|\nabla \eta|^{2} d x
$$

holds for every $H^{1}$ function $\eta=\eta(\rho)$ with compact support, and the constant $(5-2)^{2} / 4=9 / 4$ is the best possible (even among compactly supported Lipschitz functions). Hence, since

$$
4>\frac{9}{4}=\frac{(5-2)^{2}}{4}
$$


we have that the second integral in (5.5) is negative for some compactly supported Lipschitz function $\eta$. We conclude that $\bar{u}$ is unstable. Therefore, by the arguments preceding this proof, also every bounded solution of $-\Delta u=f(u)$ in $\mathbb{R}^{6}$ such that $u=0$ on the Simons cone $\mathcal{C}$ and $u$ is positive in $\mathcal{O}$ is unstable.

The following is a direct way (without using Hardy's inequality) to see that the second integral in (5.5) is negative for some Lipschitz function $\eta$ with compact support in $(0, \infty)$. For $\alpha>0$ and $0<2 \rho_{1}<1<\rho_{2}$, let

$$
\eta(\rho)= \begin{cases}\left(1-\rho_{2}^{-\alpha}\right) \rho_{1}^{-1}\left(\rho-\rho_{1}\right) & \text { for } \rho_{1} \leq \rho \leq 2 \rho_{1} \\ 1-\rho_{2}^{-\alpha} & \text { for } 2 \rho_{1} \leq \rho \leq 1 \\ \rho^{-\alpha}-\rho_{2}^{-\alpha} & \text { for } 1 \leq \rho \leq \rho_{2} \\ 0 & \text { otherwise, }\end{cases}
$$

a Lipschitz function with compact support $\left[\rho_{1}, \rho_{2}\right]$. We simply compute the second integral in (5.5) and find

$$
\begin{aligned}
\int_{0}^{+\infty} \rho^{4}\left\{\eta_{\rho}^{2}-\frac{4 \eta^{2}}{\rho^{2}}\right\} d \rho \leq & \int_{\rho_{1}}^{2 \rho_{1}} \rho^{4}\left(1-\rho_{2}^{-\alpha}\right)^{2} \rho_{1}^{-2} d \rho+ \\
& +\int_{1}^{+\infty} \alpha^{2} \rho^{2-2 \alpha} d \rho-\int_{1}^{\rho_{2}} 4 \rho^{2}\left(\rho^{-\alpha}-\rho_{2}^{-\alpha}\right)^{2} d \rho
\end{aligned}
$$

Choosing $3 / 2<\alpha<2$, as $\rho_{2} \rightarrow \infty$ the difference of the last two integrals converges to a negative number, since $\alpha^{2}<4$ and $2-2 \alpha<-1$. Since the first of the three last integrals is bounded by $7 \rho_{1}^{3}$, we conclude that the above expression is negative by choosing $\rho_{2}$ large enough and then $\rho_{1}$ small enough.

The previous proof of instability also leads to the instability outside of every compact set - and thus to the infinite Morse index property of $u$. Indeed, choosing $\rho_{1}$ and $\rho_{2}$ (and thus $\eta$ ) as above, we consider the corresponding function $\xi_{a}$ for $a>1$. Now, (5.2) and (5.5) lead to $Q_{u}\left(\xi_{a}\right) \leq Q_{\bar{u}}\left(\xi_{a}\right)<0$ for $a$ large enough. Thus, the Lipschitz function $\xi_{a}$ makes $Q_{u}$ negative for $a$ large, and has compact support contained in $\left\{a \rho_{1} \leq(s+t) / \sqrt{2} \leq a \rho_{2}\right\}$. By approximation, the same is true for a function $\xi$ of class $C^{1}$, not only Lipschitz. Hence, given any compact set $K$ of $\mathbb{R}^{6}$, by taking $a$ large enough we conclude that $u$ is unstable outside $K$, as stated in the theorem.

From the instability outside every compact set, it follows that $u$ has infinite Morse index in the sense of Definition 1.8. Indeed, let $X_{k}$ be a subspace of $C_{c}^{1}\left(\mathbb{R}^{6}\right)$ of dimension $k$, generated by functions $\xi_{1}, \ldots, \xi_{k}$, and with $Q_{u}(\xi)<0$ for all $\xi \in X_{k} \backslash\{0\}$. Let $K$ be a compact set containing the support of all the functions $\xi_{1}, \ldots, \xi_{k}$. Since $u$ is unstable outside $K$, there is a $C^{1}$ function $\xi_{k+1}$ with compact support in $\mathbb{R}^{6} \backslash K$ for which $Q_{u}\left(\xi_{k+1}\right)<0$. Since $\xi_{k+1}$ has disjoint support with each of the functions $\xi_{1}, \ldots, \xi_{k}$, it follows that $\xi_{1}, \ldots, \xi_{k}, \xi_{k+1}$ are linearly independent and that $Q_{u}\left(a_{1} \xi_{1}+\cdots+a_{k+1} \xi_{k+1}\right)=Q_{u}\left(a_{1} \xi_{1}+\cdots+a_{k} \xi_{k}\right)+Q_{u}\left(a_{k+1} \xi_{k+1}\right)<0$ for every nonzero linear combination $a_{1} \xi_{1}+\cdots+a_{k+1} \xi_{k+1}$ of them. We conclude that $u$ has infinite Morse index. 


\section{Asymptotic Stability of $\bar{u}$ In Dimensions $2 m \geq 8$}

In [14 we proved that the argument used to prove instability in dimension 4 could not be used in higher dimensions (see also the begining of the previous section). It is natural to check whether the computations we used in the proof of the previous theorem in dimension 6 lead to a instability result in higher dimensions. This is not the case, even in dimension 8 . This is an indication that saddle solutions might be stable in dimension 8 or higher.

We recall the computations in the proof of the previous theorem. Using the linearized equation (5.3) and $\xi_{a}=\xi=\eta(y / a) \bar{u}_{z}(y, z)=\eta(\rho) \bar{u}_{z}(a \rho, z)$, we reached in (5.4)

$$
\begin{gathered}
c_{m} Q_{\bar{u}}\left(\xi_{a}\right)=a^{2 m-3} \int_{\{-a \rho<z<a \rho\}} \rho^{2(m-1)}\left(1-\frac{z^{2}}{a^{2} \rho^{2}}\right)^{m-1}\left(\eta_{\rho}^{2} \psi^{2} \bar{u}_{z}^{2}-\right. \\
\left.-\eta^{2}\left\{\frac{2(m-1)\left(1+\frac{z^{2}}{a^{2} \rho^{2}}\right)}{\rho^{2}\left(1-\frac{z^{2}}{a^{2} \rho^{2}}\right)^{2}} \bar{u}_{z}^{2}-\frac{4(m-1) z}{a \rho^{3}\left(1-\frac{z^{2}}{a^{2} \rho^{2}}\right)^{2}} \bar{u}_{y} \bar{u}_{z}\right\}\right) d \rho d z,
\end{gathered}
$$

where $\eta=\eta(\rho)$ has compact support $\left[\rho_{1}, \rho_{2}\right] \subset(0, \infty)$ and $\bar{u}_{y}, \bar{u}_{z}$ are evaluated at $(a \rho, z)$. Dividing by $a^{2 m-3}$, we obtain

$$
\begin{aligned}
& \frac{c_{m} Q \bar{u}\left(\xi_{a}\right)}{a^{2 m-3}}= \\
& =\int_{\{-a \rho<z<a \rho\}} \rho^{2(m-1)}\left(1-\frac{z^{2}}{a^{2} \rho^{2}}\right)^{m-1} \bar{u}_{z}^{2} \cdot \\
& \cdot\left\{\eta_{\rho}^{2}-\frac{2(m-1)}{\rho^{2}} \frac{\left(1+\frac{z^{2}}{a^{2} \rho^{2}}\right)}{\left(1-\frac{z^{2}}{a^{2} \rho^{2}}\right)^{2}} \eta^{2}\right) d \rho d z \\
& +\int_{\{-a \rho<z<a \rho\}} \frac{4(m-1) z}{a} \rho^{2 m-5}\left(1-\frac{z^{2}}{a^{2} \rho^{2}}\right)^{m-3} \bar{u}_{y} \bar{u}_{z} d \rho d z .
\end{aligned}
$$

Using $\bar{u}_{z} \geq 0$ in $\mathbb{R}^{2 m}$ and the asymptotic limit of $\left|\bar{u}_{y}\right|$ as in the proof of instability in dimension 6 (see the previous section), the integrals above lead to

$$
\limsup _{a \rightarrow \infty} \frac{c_{m} Q_{\bar{u}}\left(\xi_{a}\right)}{a^{2 m-3}}=\left\{\int_{-\infty}^{+\infty} \dot{u}_{0}^{2}(z) d z\right\} \int \rho^{2(m-1)}\left(\eta_{\rho}^{2}-\frac{2(m-1)}{\rho^{2}} \eta^{2}\right) d \rho .
$$

The second integral may be thought as an integral of a radial function in $\mathbb{R}^{2 m-1}$. Using Hardy's inequality (see e.g. [21]) we have that this integral is nonnegative for all Lipschitz $\eta$ if and only if

$$
2(m-1) \leq \frac{(2 m-1-2)^{2}}{4}
$$

Writting $n=2 m$, the above inequality holds if and only if

$$
n^{2}-10 n+17 \geq 0,
$$

that is, $n \geq 8$. Thus, for $n \geq 8$ the inequality is true (it is even strict) and we conclude some kind of asymptotic stability of $\bar{u}$. 


\section{REFERENCES}

[1] S. Alama, L. Bronsard, and C. Gui, Stationary layered solutions in $\mathbb{R}^{2}$ for an AllenCahn system with multiple well potential, Calc. Var. Partial Differential Equations 5 (1997), 359-390.

[2] G. Alberti, L. Ambrosio, and X. Cabré, On a long-standing conjecture of E. De Giorgi: symmetry in $3 D$ for general nonlinearities and a local minimality property, Acta Appl. Math. 65 (2001), 9-33.

[3] H. Alencar, A. Barros, O. Palmas, J. G. Reyes, and W. Santos, $\mathrm{O}(\mathrm{m}) \times \mathrm{O}(\mathrm{n})$-invariant minimal hypersurfaces in $\mathbb{R}^{m+n}$, Ann. Global Anal. Geom. 27 (2005), 179-199.

[4] F. Alessio, A. Calamai, and P. Montecchiari, Saddle-type solutions for a class of semilinear elliptic equations, Adv. Differential Equations 12 (2007), 361-380.

[5] L. Ambrosio and X. Cabré, Entire solutions of semilinear elliptic equations in $\mathbb{R}^{3}$ and a conjecture of De Giorgi, Journal Amer. Math. Soc. 13 (2000), 725-739.

[6] S. B. Angenent, Uniqueness of the solution of a semilinear boundary value problem, Math. Ann. 272 (1985), 129-138.

[7] D. G. Aronson and H. F. Weinberger, Multidimensional nonlinear diffusion arising in population genetics, Adv. in Math. 30 (1978), 33-76.

[8] H. Berestycki, L. Caffarelli, and L. Nirenberg, L. Monotonicity for elliptic equations in unbounded Lipschitz domains, Comm. Pure Appl. Math. 50 (1997), 1089-1111.

[9] H. Berestycki, L. Caffarelli, and L. Nirenberg, Further qualitative properties for elliptic equations in unbounded domains, Ann. Scuola Norm. Sup. Pisa Cl. Sci. 25 (1997), 69-94.

[10] H. Berestycki, F. Hamel, and N. Nadirashvili, The speed of propagation for KPP type problems. I. Periodic framework, J. Eur. Math. Soc.,7 (2005), 173-213.

[11] H. Berestycki, F. Hamel, and L. Rossi, Louville type results for semilinear elliptic equations in unbounded domains, Ann. Mat. Pura Appl. (4) 186 (2007), 469-507.

[12] H. Berestycki, L. Nirenberg, and S.R.S. Varadhan, The principal eigenvalue and maximum principle for second-order elliptic operators in general domains, Comm. Pure Appl. Math. 47 (1994), 47-92.

[13] E. Bombieri, E. De Giorgi, and E. Giusti, Minimal cones and the Bernstein problem, Inv. Math. 7 (1969), 243-268.

[14] X. Cabré and J. Terra, Saddle-shaped solutions of bistable diffusion equations in all of $\mathbb{R}^{2 m}$, to appear on J. Eur. Math. Soc.; arXiv:0801.3379.

[15] L. Caffarelli, D. Jerison, and C. Kenig, Global energy minimizers for free boundary problems and full regularity in three dimensions, Contemp. Math. 350, 83-97, Amer. Math. Soc., Providence, RI, 2004.

[16] H. Dang, P. C. Fife, and L. A. Peletier, Saddle solutions of the bistable diffusion equation, Z. Angew Math. Phys. 43 (1992), no 6, 984-998.

[17] E. De Giorgi, Convergence problems for functionals and operators, Proc. Int. Meeting on Recent Methods in Nonlinear Analysis (Rome, 1978), 131-188, Pitagora, Bologna (1979).

[18] G. De Philippis and E. Paolini, A short proof of the minimality of Simons cone, to appear in Rend. Sem. Mat. Univ. Padova, cvgmt.sns.it

[19] D. De Silva and D. Jerison, A singular energy minimizing free boundary, to appear in J. Reine Angew. Mat.

[20] M. del Pino, M. Kowalczyk, and J. Wei, On De Giorgi conjecture in dimension $N \geq 9$, arXiv:0806.3141

[21] J.P. García Azorero and I. Peral, Hardy inequalities and some critical elliptic and parabolic problems, J. Differential Equations 144 (1998), 441-476. 
[22] N. Ghoussoub and C. Gui, On a conjecture of De Giorgi and some related problems, Math. Ann. 311 (1998), 481-491.

[23] E. Giusti, Minimal Surfaces and Functions of Bounded Variation, Birkhäuser Verlag, Basel-Boston, 1984.

[24] D. Jerison and R. Monneau, The existence of a symmetric global minimizer on $\mathbb{R}^{n-1}$ implies the existence of a counter-example to a conjecture of De Giorgi in $\mathbb{R}^{n}$, C. R. Acad. Sci. Paris, Ser. I 333 (2001), 427-431.

[25] L. Modica, A gradient bound and a Liouville theorem for nonlinear Poisson equations, Comm. Pure Appl. Math. 38 (1985), 679-684.

[26] O. Savin, Regularity of flat level sets in phase transitions, Ann. Math. 169 (2009), 41-78.

[27] M. Schatzman, On the stability of the saddle solution of Allen-Cahn's equation, Proc. Roy. Soc. Edinburgh Sect. A 125 (1995), no. 6, 1241-1275.

[28] J. Simons, Minimal varieties in riemannian manifolds, Ann. Math. 88 (1968), 62105.

ICREA and Universitat Politècnica de Catalunya, Departament de Matemàtica Aplicada I, Diagonal 647, 08028 Barcelona, Spain

E-mail address: xavier.cabre@upc.edu

Universidad de Buenos Aires, Departamento de Matemática, Ciudad Universitaria, Buenos Aires, Argentina

E-mail address: jterra@dm.uba.ar 